JoURnAL OF

SEPARATION

$1 \mid 2021$

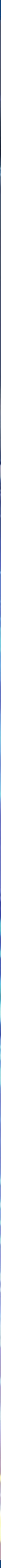

Chromatography · Electroseparation 


\section{The role of sample preparation in multidimensional gas chromatographic separations for non-targeted analysis with the focus on recent biomedical, food, and plant applications}

\author{
Flavio A. Franchina $\odot$ \\ Jean-François Focant
}

\author{
Delphine Zanella $₫ \quad$ Lena M. Dubois
}

Molecular System, Organic \& Biological Analytical Chemistry Group, University of Liège, Liège, Belgium

\section{Correspondence}

Flavio A. Franchina, PhD, Molecular System, Organic \& Biological Analytical Chemistry Group, University of Liège, Liège, Belgium.

Email: flaviofranchina@gmail.com; ffranchina@uliege.be

This review article is intended for publication in the Special Issue "Reviews 2021".

\section{Funding information}

Fonds De La Recherche Scientifique FNRS, Grant/Award Number: 30897864; Fonds Wetenschappelijk Onderzoek, Grant/Award Number: 30897864; Fonds pour la Formation à la Recherche dans l'Industrie et dans l'Agriculture
In this review, we consider and discuss the affinity and complementarity between a generic sample preparation technique and the comprehensive twodimensional gas chromatography process. From the initial technical development focus (e.g., on the $\mathrm{GC} \times \mathrm{GC}$ and solid-phase microextraction techniques), the trend is inevitably shifting toward more applied challenges, and therefore, the preparation of the sample should be carefully considered in any GC $\times \mathrm{GC}$ separation for an overreaching research. We highlight recent biomedical, food, and plant applications (2016-July 2020), and specifically those in which the combination of tailored sample preparation methods and $\mathrm{GC} \times \mathrm{GC}-\mathrm{MS}$ has proven to be beneficial in the challenging aspects of non-targeted analysis. Specifically on the sample preparation, we report on gas-phase, solid-phase, and liquid-phase extractions, and derivatization procedures that have been used to extract and prepare volatile and semi-volatile metabolites for the successive $\mathrm{GC} \times \mathrm{GC}$ analysis. Moreover, we also present a milestone section reporting the early works that pioneered the combination of sample preparation techniques with $\mathrm{GC} \times \mathrm{GC}$ for non-targeted analysis.

\section{KEYWORDS}

comprehensive two-dimensional gas chromatography, mass spectrometry, metabolomics, sample extraction

\begin{abstract}
Abbreviations: CAR, carboxen; CBD, cannabidiol; CBN, cannabinol; CSC, coconut shell charcoal; DHS, dynamic headspace; DI, direct immersion; DVB, divinylbenzene; EG, ethyleneglycol; FID, flame ionization detector; FM, flow modulation; GC-GC, heartcutting multidimensional gas chromatography; GPe, gas-phase extractions; HR, high resolution; HS, headspace; HSSE, headspace sorptive extraction; LPe, liquid-phase extractions; MDE, magnetic dispersive extraction; MSTFA, N-Trimethylsilyl-N-methyl trifluoroacetamide; NTME, needle trap microextraction; ODS/GC, octa-decyl silica and graphite carbon; P\&T, purge and trap; PBS, phosphate-buffered saline; PDMS, polydimethylsiloxane; PIL, poly ionic-liquid; PLE, pressurized liquid extraction; QTOF, quadrupole-time of flight mass analyzer; SBSE, stir
\end{abstract}

\section{1 | INTRODUCTION}

The first decade after the seminal paper from Phillips [1] was especially abundant in the research for a better understanding of the $\mathrm{GC} \times \mathrm{GC}$ process and orthogonality, the visualization of the data, and the surprising "rediscovery" of samples' composition. These, in addition

\footnotetext{
bar sorptive extraction; SDE, simultaneous distillation extraction; SFE, supercritical fluid extraction; SHS, static headspace; SPe, solid-phase extractions; SVOC, semi volatile organic compound; Tenax TA, 2,6-diphenyl-p-phenylene oxide; THC, tetrahydrocannabinol; VOC, volatile organic compound; XAD-2, styrene-divinylbenzene resin.
} 
Sample preparation

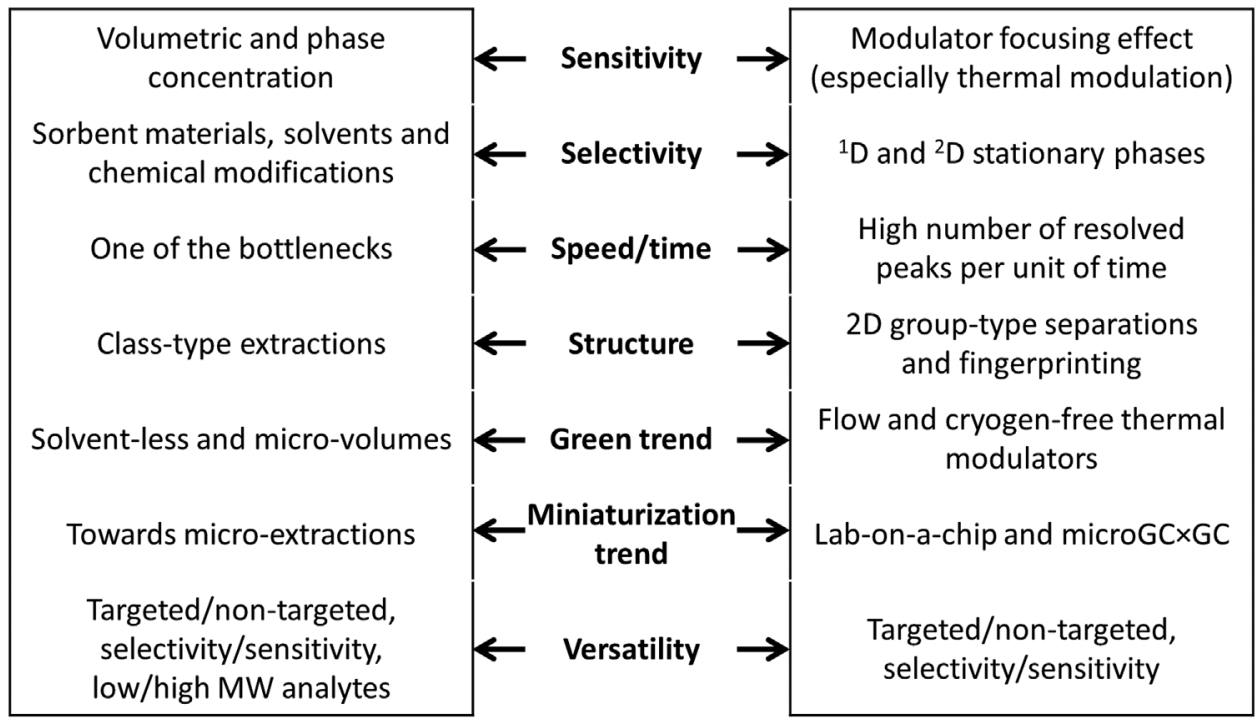

F I G U R E 1 Commonalities and characteristics of a generic sample preparation and the GC $\times$ GC separation steps

to the advancements of the transfer process, which also led to the development of the different modulation forms available nowadays, represented the main playground for researchers in the early stage of GC $\times \mathrm{GC}$ [2].

The sample preparation initially retained little attention within $\mathrm{GC} \times \mathrm{GC}$ studies, with extremely limited or basic procedures, as already pointed out [3], and with the main attention focused around the novelty of the multidimensional separation concept. The minor consideration for the sample preparation when combined with GC $\times \mathrm{GC}$ was also partially due to the additional features of the multidimensional technique (increased selectivity, sensitivity, speed, separation power, structure), compared to the conventional (1D) GC separation. Nevertheless, even if the additional 2D separation helps with analytes of interest enrichment (i.e., the focusing effect especially for thermal modulators), cleanup, and interference removal (fewer coelutions, and separation from the chemical noise, i.e., columns bleed), it does not justify overlooking a proper and optimized sample preparation method. The preparation and extraction of the analytes of interest indeed represent the most crucial step in the analytical workflow as it should provide an authentic representation of the sample, setting a solid basis for the following analytical stages [4].

The introduction of SPME by Pawliszyn represented the other groundbreaking advance which contributed strongly to the increase of the consideration and use of novel, green, and miniaturized sample preparation techniques with $\mathrm{GC} \times \mathrm{GC}[5,6]$. The most used form of SPME consists of fused silica fibers coated with the extraction phase(s), which are exposed to the headspace of, immersed in, or put in direct contact with the sample [4].
It is worthy to highlight the fact that $\mathrm{GC} \times \mathrm{GC}$ and SPME originated (1) in the same period [1,7], (2) from two researchers who shared the same research laboratory for some time, and (3) from similar fundamental concepts; these facts are signs of the intimate relation of the two techniques. Figure 1 resumes some of the key features of a generic sample preparation and the $\mathrm{GC} \times \mathrm{GC}$ separation, highlighting the commonalities between the two processes. Shared features and attributes can be identified for both the sample preparation and the standalone $\mathrm{GC} \times \mathrm{GC}$ process. Among these, the enrichment and clean-up of analytes of interest, the interference removal, the tuning of the selectivity, and the versatility of the approaches make them the most powerful analytical alliance for complex samples analysis. This becomes exceptionally true when the detection is represented by mass spectrometry, thanks to the unique identification capabilities to handle a multitude of the extracted and separated analytes. In this case, the data generated intrinsically contain all the information for applying either a fingerprinting, a profiling, and/or a targeted approach, based on the research questions.

An additional and common point regards the trend toward greener approaches and miniaturization which is true for both sample preparation and the GC $\times \mathrm{GC}$ separation $[3,5]$.

All the new evolutions after the introduction of SPME and $\mathrm{GC} \times \mathrm{GC}$, together with the rapid development of MS and software-data handling tools since early 2000 , surely ignited the growing and valued the use of non-targeted analysis in all applications fields. Nowadays, it is possible to find demonstrations of the usefulness of non-targeted analysis combining the many available forms of sample 
preparation with the high-resolution GC $\times$ GC-MS technique. In this review, we report on gas-phase (GPe), solidphase (SPe), liquid-phase (LPe) extractions, and derivatization procedures which have been used to extract and analyze volatile (boiling point range between 50 and $260^{\circ} \mathrm{C}$ ) and semi-volatile (boiling point range between $240-400^{\circ} \mathrm{C}$ ) metabolites from biological, food and plant samples, with the focus on non-targeted applications in last 5-years period (2016-July 2020). The description of the underlying mechanism of the extraction types (i.e., GPe, $\mathrm{SPe}, \mathrm{LPe}$ ) is reported in the following paragraph 3.

\section{2 | MILESTONES IN COMBINING SAMPLE PREPARATION TECHNIQUES AND GC $\times$ GC}

Thirty years have passed since the first report of the modern sample preparation SPME and comprehensive 2D-GC techniques. The intent of this paragraph is to recognize the early contributions that pioneered the combination of sample preparation with high-resolution separation and detection for non-targeted analysis in biological, food, and plant applications. It must be said that these early works that initiated the use of innovative sample preparation techniques as up-front tools for $\mathrm{GC} \times \mathrm{GC}$, yet focused mainly on the increase of selectivity and sensitivity of the multidimensional separation technique.

SPe techniques were the earliest sample preparation procedures used with $\mathrm{GC} \times \mathrm{GC}$. In 2002, Adahchour et al. evaluated the general practicability of combining headspace (HS)-SPME with GCXGC for the analysis of flavors in garlic cloves [8]. Compared to conventional GC, a 10- to 50-fold increase in sensitivity together with a 10-times higher peak capacity were reported. In addition, the comparison of the PDMS and DVB/CAR/PDMS fibers highlighted the improved extraction efficiency (up to 20 -fold) when using the latter. Their study opened the way for HS-SPME-GC $\times \mathrm{GC}$ for detailed flavor analysis and identification of aroma active compounds. In the same year, Perera et al. reported increased sensitivity and improved peak capacity using HS-SPME and GC $\times$ GC for the analysis of wounded plants [9]. Furthermore, they highlighted the usefulness of the fingerprinting approach to define chromatographic regions of interest enabling direct and easy sample comparison. Worth mentioning, even if not relevant with the applications' fields of this review, is the very first HS-SPME-GC $\times$ GC application carried out by Frisinger et al. in 1998 for environmental volatile organic compound (VOC) determination. The authors were still using the first thermal sweeper modulator and they discussed about the special care and the details for the (manual) SPME sampling and injec- tion procedures since no automation was yet available [10].

In 2003, GC $\times \mathrm{GC}$ followed mixed-bed SPE cartridges to analyze drugs in horse and dog urine [11]. These cartridges represent the most classical SPe, and are short disposable columns containing a bed of porous particles, in which the analytes or the fractions of interest are retained after a first solvent elution, and then re-mobilized by elution using a different solvent [12]. The authors performed the derivatization of the amino groups of the drug metabolites to produce thermally stable analytes. This study demonstrated the potential of the overall methodology in drug analysis by providing lower detection limits of $0.04 \mathrm{mg} / \mathrm{L}$ against $0.1 \mathrm{mg} / \mathrm{L}$ using classical 1D GC, good reproducibility, and the possibility of high-throughput sample screening using the $2 \mathrm{D}$ chromatographic space.

In 2002, Seeley et al. employed sorbent traps with $\mathrm{GC} \times \mathrm{GC}$, equipped with a differential flow modulator and dual secondary columns, to characterize exhaled breath from healthy humans [13]. Multi-bed sorbent trap tubes (Carbotrap C/Carbotrap/Carbosieve SIII) were used to collect $1.5 \mathrm{~L}$ of breath. The system enabled the characterization of 100 compounds over a $10 \mathrm{~min}$ analysis. The unique selectivity of the two secondary columns allowed increasing further the resolution and the qualitative information provided by $\mathrm{GC} \times \mathrm{GC}$ analysis. Even though this contribution was mainly focused on the characteristics of the flow modulated system, it demonstrated the effectiveness of $\mathrm{GC} \times \mathrm{GC}$ for breath analysis, which was further investigated in the following years [14]. An alternative and interesting approach for breath analysis was highlighted in 2010 by Mieth et al., with the use of a needle trap microextraction (NTME) device [15]. Alveolar breath samples were taken from patients undergoing cardiac surgery showing the potential of the device for on-site sampling and pre-concentration, for biomarkers discovery. Good storage capabilities, up to one day according to the physicochemical properties of the analytes, and high throughput analysis using an optimized autosampler were described.

In 2012, Risticevic et al. investigated for the first time the potential of direct immersion (DI)-SPME and GC $\times$ GC for metabolite profiling of apples [16]. An increased (351 supplementary metabolites) and more balanced metabolite coverage were obtained when using DI-SPME, avoiding loss of polar and high molecular weight analytes. However, it was highlighted that a comprehensive characterization of the metabolome was only achievable under optimized conditions for both the sample preparation and the separation. The authors fully exploited GC $\times \mathrm{GC}$ attributes, i.e., the $2 \mathrm{D}$ separation space occupation and the structured separation, to optimize the SPME method.

Cordero et al. also took advantage of multiple sampling techniques with the GCXGC attributes [17]. The 
complementarity of gas-phase (i.e., dynamic headspace (DHS)) and solid-phase (i.e., headspace sorptive extraction (HSSE), HS-SPME, DI-SPME, and stir bar sorptive extraction (SBSE)) sampling approaches was demonstrated in the characterization of VOCs and semi-volatile organic compounds (SVOCs) in dry milk powder. In DHS, continuous removal of volatiles from the matrix is obtained with a constant inert gas flow to increase the efficiency of the extraction. Regarding SBSE, it has the same working principles of SPME: in this case, a magnetic stir bar is coated with the sorbent and can be immersed (classical SBSE), or suspended in the headspace (HSSE) of the samples. The authors highlighted for the first time the effectiveness of the combination of HSSE and SBSE with GC $\times$ GC for a sensomics investigation in food analysis thanks to their high concentration factors enabling broad analyte coverage. These results pointed out the importance of the choice of the sample preparation since this latter can greatly influence the obtained fingerprints of complex volatile fractions.

Shortly after its introduction, GC $\times$ GC-MS also served the emerging metabolomics field. The great potential of structured 2D chromatograms has been initially shown for lipids $[18,19]$. In these early reports, a derivatization procedure (i.e., transesterification) was necessary to isolate the fatty acids from the rest of the matrix and make them more suitable for GCXGC analysis, as methyl ester derivatives. The modulator band compression allowed the concurrent determination of the major as well as minor components of milk and various vegetable and fish oils, revealing a novel chemical complexity of those "well-known" samples.

Following such a demonstration of the increased capacities of GC $\times$ GC over $1 D$ GC, in $2005 \mathrm{GC} \times \mathrm{GC}$ was used to identify biomarkers of obesity extracted from mouse spleen [20]. The extracted metabolites were derivatized with commonly used silylation agents, prior to $\mathrm{GC} \times \mathrm{GC}$ analysis. Amino- and hydroxyl acids together with few carbohydrates' derivatives were identified in the two populations, with sugar alcohols classified as potential biomarkers of obesity. This was the first claimed non-targeted metabolomics study using GCXGC-MS, demonstrating that direct translation from 1D GC-MS to 2D GC-MS was possible, and set the basis for the use of GC $\times \mathrm{GC}$ in biomedical research.

\section{3 | NON-TARGETED ANALYSIS APPLICATIONS}

With the refinement and advancement of the hardware, which improves the technique robustness and reliability, and software tools, which help with the high-dimensional data handling and processing, recent applications are more and more focused on applied challenges. The workflow used for the literature survey in the period covering 2016 until mid-2020 is provided in the supporting information (Supporting Information Figure S1). These references are listed in Supporting Information Table S1, which also contains brief information on the sample type, the sample preparation, and the GC $\times$ GC-MS methodology. Selected biomedical-, food-, and plant-related applications which reported more emphasis on the sampling step are instead described within each of the following section to summarize the recent trends and challenges of non-targeted analysis combining innovative sample preparation techniques and GCXGC. Unless otherwise stated, all the applications described in this review involved GC $\times$ GC-MS with cryogenic modulation. Some remarkable applications during the covered period are summarized in Table 1, in which more technical details on the sample preparation conditions are reported.

Along the review, the modern sample preparation techniques which preceded the GC $\times$ GC-MS analysis were grouped into the principal classifications of GPe, SPe, LPe, and derivatization protocols (Figure 2) [21].

In $\mathrm{GPe}$, a gas is used to strip the volatile compounds off the sample, which can be injected directly (traditional static HS) or trapped onto a sorbent trap. SPe is based on the transfer of the compounds of interest from a gas, liquid, or supercritical fluid matrix to a solid sorbent. In LPe, the extracting agent is a liquid fluid, as in the common case of liquid-liquid extractions [22].

SPe and most of the GPe techniques rely on sorbentbased interactions with the analytes to extract. Among these techniques, surely SPE, SPME, SBSE, NTME, and DHS through trap tubes are the most promising and the most used with GC $\times$ GC. For a more detailed description of the techniques reported in this review, please refer to the literature $[12,23,24]$.

Generally, GPe methods are exclusively dedicated to VOC sampling, while SPe, LPe, and the derivatization approaches are suitable also for the heavier SVOCs. Among $\mathrm{SPe}$, some techniques can be used both in headspace or direct immersion approaches (e.g., HS/DI-SPME and HSSE/SBSE) [25]. In the case of multistep sample preparation protocols, for example, a derivatization step followed by multiple extractions, the sample preparation techniques were counted separately for the generation of Figure 2 .

As already highlighted, each analytical step and especially the sample preparation could introduce a bias (in the selectivity) in the final result, which makes the realization of a true non-targeted and unbiased analysis very challenging and arguable. For example, any extraction strategy sets at the inception of the whole methodology a certain degree of selectivity towards a defined class of analytes, depending on their chemical 


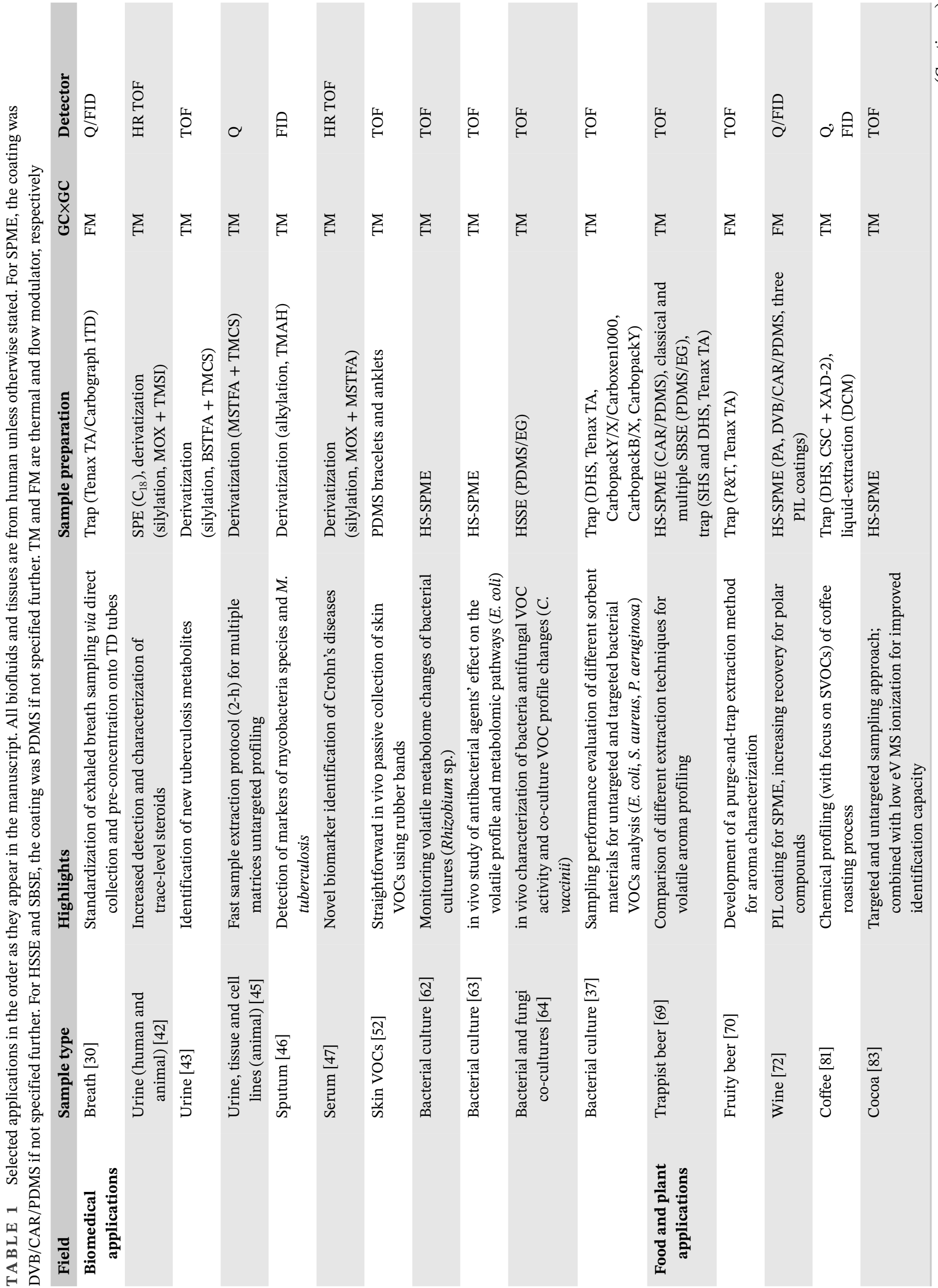


or physical properties. Keeping in mind this unavoidable bias-introduction, non-targeted strategies generally tend to use wide-selectivity analytical steps to characterize in-depth the samples, track and/or compare hundreds of analytes between groups of samples to address specific research questions. Like for the GC column chemistries, different materials with variable selectivity are available for the sorbent-based extractions. The discussion on the different sorbent materials and their selectivity will not be herein described, and a series of reviews and books are provided for more detailed information [12,23,24,26].

Over this period of 5 years (2016-2020), we observed that the characterization of volatile metabolites from biological, food, and plant samples through headspace sampling represents the most common approach $(57 \%)$ used in combination with $\mathrm{GC} \times \mathrm{GC}$. A dominant position for sampling is held by SPME, which appears to be used preferably in the HS mode (39\%) rather than in DI mode (1\%).

\section{1 | Biomedical-related}

The interest for non-targeted metabolomic analysis in biomedical research is in constant development, making it one of the most active and promising fields of application. Studies are mainly driven by the search for novel diagnostic and prognostic biomarkers of heath-altered states, and their basis relies on the fact that metabolite levels vary at the early stage of the diseases. Early diagnosis together with an increased understanding of human diseases would allow improving the quality of life and expectations of the patients. However, there are numerous challenges associated with biomarker discovery in this field. In particular, the variability and complexity of the biological matrices (i.e., biological fluids, biopsies, tissues, cell cultures), comprising thousands of metabolites characterized by a wide range of concentrations and physicochemical properties, are posing the major challenges. In addition, a single analytical platform is not sufficient to exhaustively cover and analyze such a broad range of metabolites, and thus multiplatform strategies should ideally be sought [27]. The diversity of the metabolome and the related difficulties to identify de novo metabolites delay the direct correlation or mapping of (novel) biochemical pathways [28].

In view of the sample preparation techniques used over the past 5 years, HS-SPME together with sorbent traps and derivatization procedures have been the most used sampling techniques, representing together $\sim 70 \%$ of the methods reported in the 2016-2020 literature (Figure 2). 


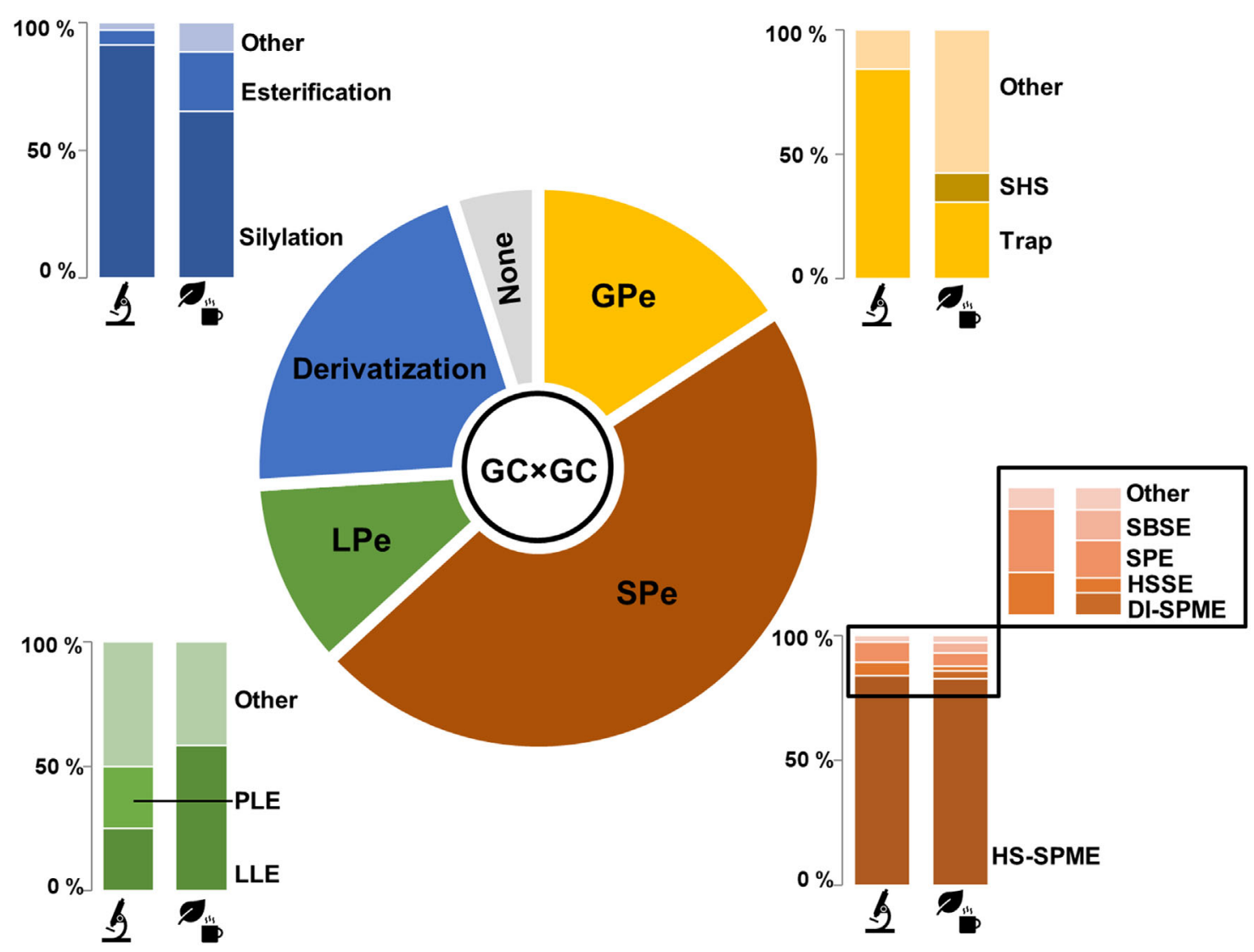

F I G U R E 2 Distribution of the sample preparation types used in combination with GC $\times$ GC in the period 2016-2020. In the bar charts, the bar on the left groups biomedical-related applications, and the one on the right groups the food- and plant-related applications

\subsection{1 | Biological fluids}

Blood, urine, and exhaled breath have been thoroughly studied matrices to identify potential biomarkers of metabolism perturbations $[29,30]$.

The growing interest for exhaled breath analysis can be explained by its non-invasive nature, allowing its use for a broad range of patients. Exhaled breath sampling for $\mathrm{GC} \times \mathrm{GC}$ analysis is a two-stage process since the direct injection into the separation system has not yet been demonstrated. In most of the studies, breath collection was performed using Tedlar sampling bags before the pre-concentration on sorbent traps. In this case, the pre-concentration step that enables sample enrichment is particularly important, as analytes are present in trace-levels. Over the past 5 years, trap tubes have been the most commonly used sampling technique for breath analysis [31-36], although a more recent trapping technique, the NTME has demonstrated great potential for breath analysis [37]. Ideally, NTME devices and trap tubes could be used for simultaneous collection and pre-concentration avoiding the intermediate use of Tedlar bags, but until today, only few specific devices exist [38-40]. In a recent study, Wilde et al. opti- mized the sampling parameters of a device enabling the direct collection and pre-concentration of breath, with no need for a derivatization step, onto trap tubes to identify potential markers of acute breathlessness [35]. In their study, the trap tubes were desorbed in a flowmodulated (FM)-GC $\times$ GC system with dual MS and flame ionization detection (FID), combining high-separation power, more uniform response factors, and identification capabilities.

In another work, VOC bio-sampling in serum and exhaled breath using trap tubes was evaluated among different packing materials, highlighting the importance of the sorbent selection due to the different matrix contributions (e.g., relative humidity effect on the sorbent type and complexity) [41]. In Figure 3 is shown the longitudinal sampling profile of some endogenous metabolites released in breath after coffee ingestion using differently packed tubes. Among the six tested sorbent traps, the tubes packed with Tenax TA showed the best sampling performance in terms of reproducibility and sensitivity, allowing longer metabolite tracking times and higher consistency of the measurements. The results reinforced the previous observations about the sampling for bacterial VOC (see Section 3.1.3) [42]. 


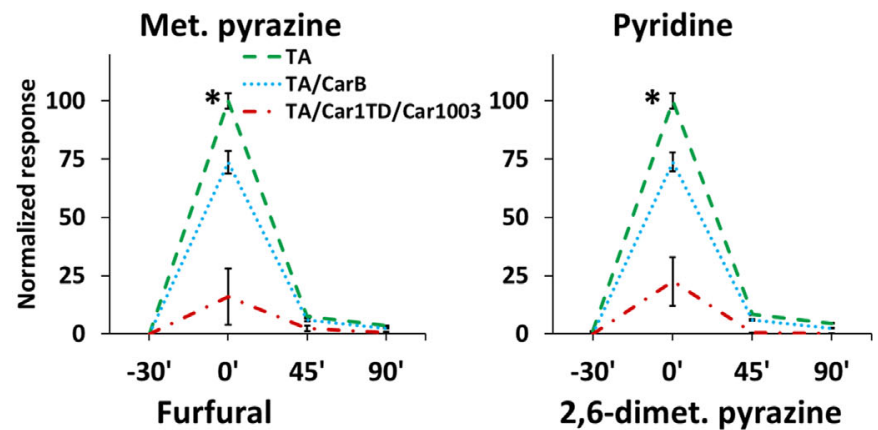

Furfuryl met. sulfide

1-met. 1H-pyrrole
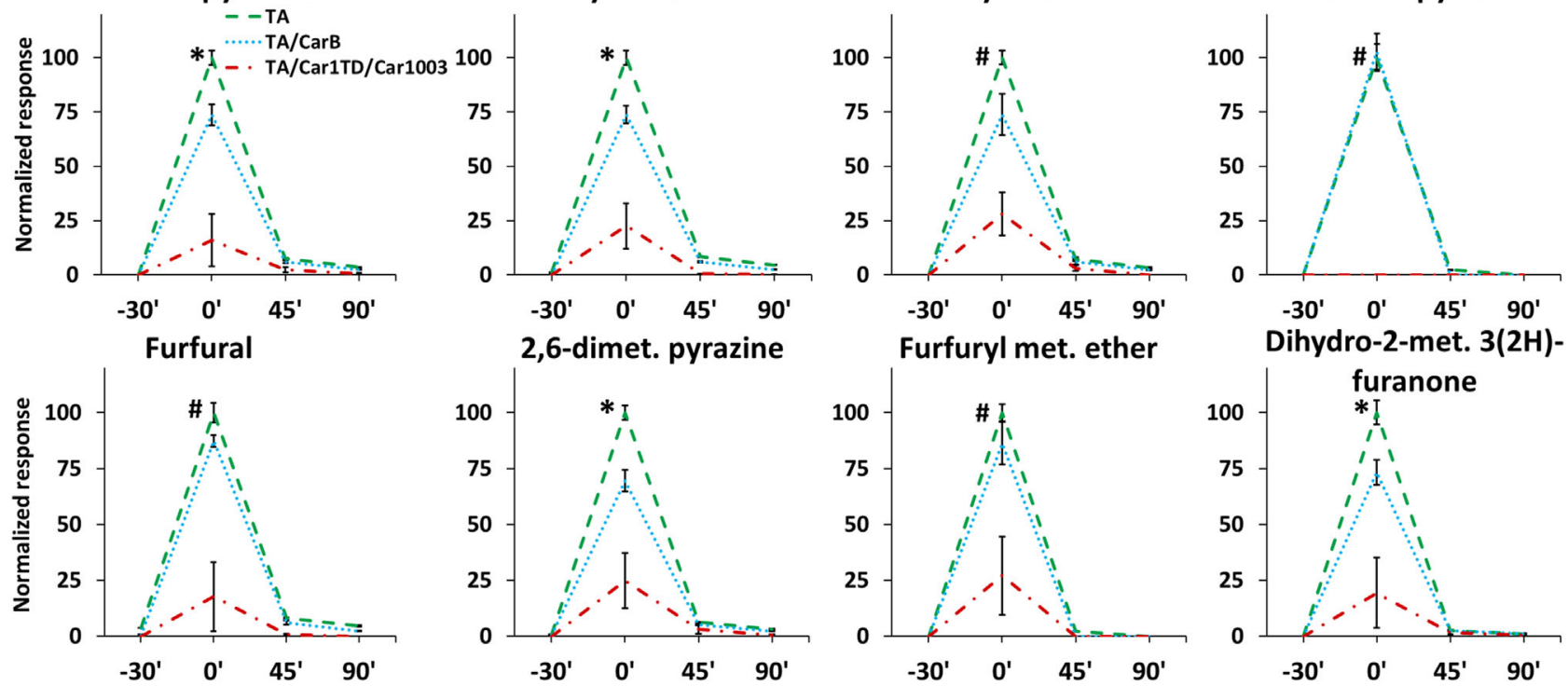

F I G U R E 3 Longitudinal tracking of breath metabolites from coffee (-30, 0, 45, and 90 min with respect to its intake) using different sorbent traps. Reproduced from [41] with permission from Elsevier

\section{DVB/CAR/PDMS}

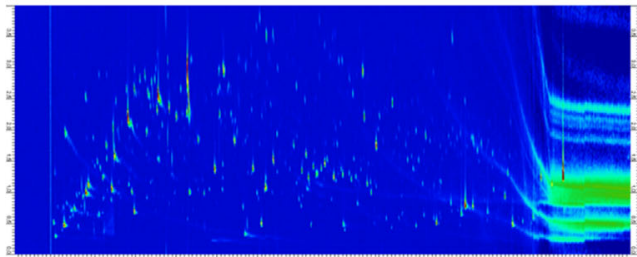

PDMS

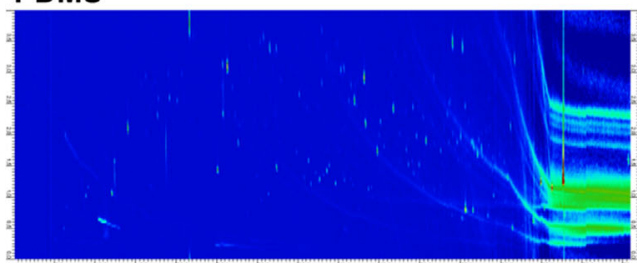

PDMS/DVB

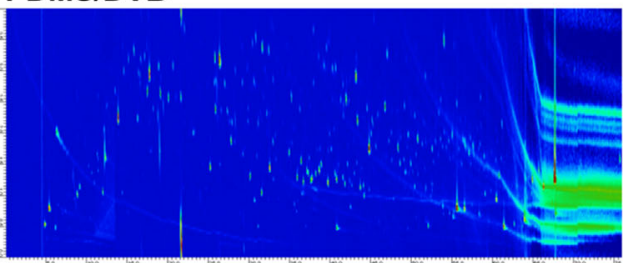

PA

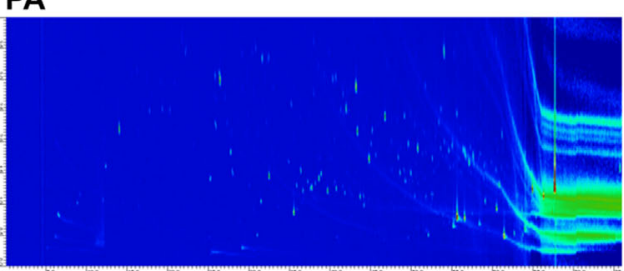

F I G U R E 4 Different HS-SPME-GC $\times$ GC-MS chromatograms of a urine sample using different fiber sorbent phases. Reproduced with permission from [43]

In the context of biofluids, SPME in HS mode is the second most popular technique for volatile analytes characterization by GC $\times \mathrm{GC}$ [43-46]. Mack et al. evaluated the efficiency of different HS-SPME fibers to measure urinary volatile metabolites linked to dietary intake [43]. As can be seen in Figure 4, a richer chromatogram and higher extraction yields were obtained when using the triphasic DVB/CAR/PDMS fiber, with the authors pointing out the importance of careful evaluation and optimization of the materials/conditions for analyte extraction. In addition, they highlighted the impact of data treatment and especially the normalization. They compared different normalization approaches relying on physiologic properties (i.e., osmolality, creatinine, and urine volume), and data-driven normalizations (i.e., mass spectral total useful signal and probabilistic quotient normalization), and among six compounds identified as potential markers of coffee consumption, only one was confirmed independently of the normalization used.

The triphasic fiber is common and appears to be an optimal fiber for biofluid analysis, and has been used to extract VOCs from urine and blood for different objectives such as asthma phenotyping [44], colorectal cancer diagnosis [45], and fertility monitoring [46].

When seeking for increased metabolite coverage, for example, high molecular weight and/or polar metabolites, 

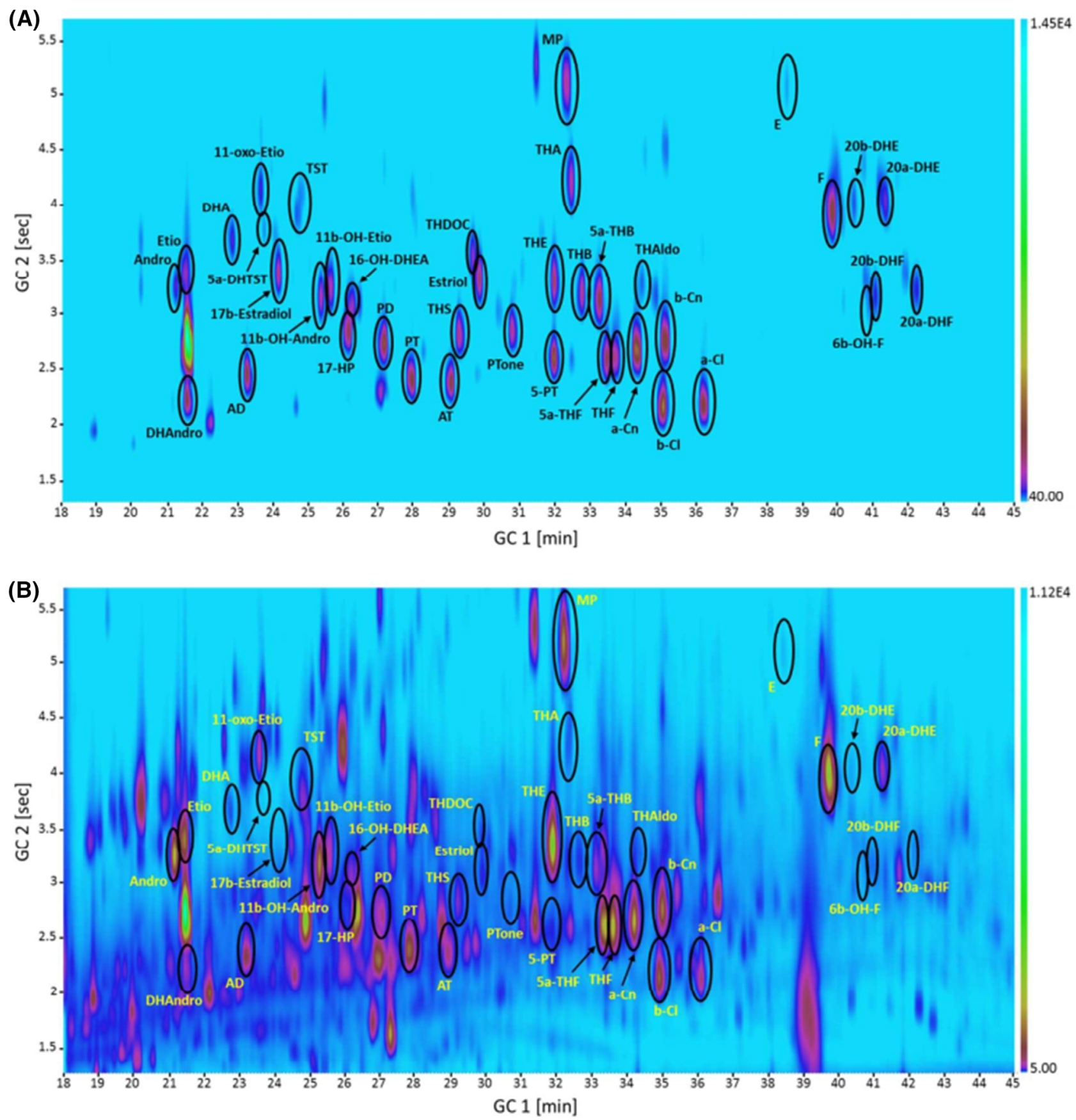

F I G U R E 5 GC $\times$ GC-MS chromatograms of derivatized (A) 40 standard steroids and two internal standards and (B) a human urine sample. Reproduced from [47] with permission from the Royal Society of Chemistry

a derivatization procedure is widely used to make sugars, amino acids, fatty acids, and steroids amenable for GC analysis and to characterize them. Such metabolites are essential to living organisms and their levels are most of the time correlated to metabolism perturbations or disease states. Even though the derivatization protocols make the sample preparation more extensive, their utilization can be maximized in combination with GC $\times$ GC [47-55].

Bileck et al. described a non-targeted method for the comprehensive analysis of steroid metabolites in urine
[47]. Using a previously optimized derivatization procedure, they highlighted the complexity of the matrix and the numerous co-elutions between urinary steroids and other metabolites (Figures 5A-5B). The increased separation power of $\mathrm{GC} \times \mathrm{GC}$ enabled the characterization of up to 70 steroids in urine from different sources (infants, adults, and rodents) against 26 using classical GC- and LC-MS methods.

Additional studies reported the increased characterization of the urine metabolome combining a well-established 
derivatization process and GC $\times$ GC separation, which enabled to discover new tuberculosis markers reflecting adaptations from the host-pathogen interaction and treatment failure $[48,49]$.

In some cases, the increased resolution provided by GC $\times$ GC was not sufficient, and specific sample preparation was necessary for the determination of specific classes of metabolites. For example, the derivatization of serum and urine samples using methoxyamine and MSTFA (1\% TMSCI) yielded in the trimethylsilane adducts of lactate and pyruvate that were not sufficiently different to be separated. Instead, the use of one-step derivatization protocol using MSTFA ( $1 \%$ TMSCI) enabled the separation of the derivatized forms of lactate and pyruvate, suggesting that variations in derivatization strategies may further increase molecular feature detection in complex biomedical samples [50].

At the cost of making the global sample preparation more extensive, Di Giovani et al. insisted on the need for careful optimization of each step of the extraction and derivatization process together with a robust experimental design to identify serological biomarkers of Crohn's diseases, and long-term quality controls [52]. Here, the optimized sample preparation procedure allowed identifying 33 potential biomarkers in serum to discriminate healthy volunteers from three subgroups of patients having Crohn's disease, namely with high, low, and quiescent endoscopic activity.

\subsection{2 | Tissue and biopsies}

When the starting material is a tissue, time-consuming multistep sample preparation is required, especially for hypothesis-generating, non-targeted metabolomics studies. Sample homogenization, metabolites extraction, and derivatization are some of the steps usually involved in the sample preparation [50,56]. Joseph et al. identified some metabolites related to lipid metabolism in rat muscle which could be used in therapeutic approaches for monitoring type 2 diabetes and obesity [56]. However, the sample preparation required approximately $14 \mathrm{~h}$ of labor. Yu et al. developed and validated a sampling protocol requiring only $2 \mathrm{~h}$ for metabolite extraction and derivatization from tissue, demonstrating the potential for further improvement towards high-throughput sample preparation protocols [50].

The in vivo sampling and investigation of VOCs emitted from human skin has been recently exploited and investigated with $\mathrm{GC} \times \mathrm{GC}[57,58]$. Roodt et al. described a PDMS silicone rubber in the form of bracelets and anklets to characterize the human skin microbiome [57]. After the passive sampling for $4 \mathrm{~h}$, the bracelets were thermally desorbed into the GC $\times \mathrm{GC}$ inlet, enabling the characterization of around 300 volatiles with acceptable reproducibility ( $<40 \%$ RSD for $75 \%$ of the detected compounds). To analyze the heavier SVOCs from human skin, Dolezal et al. reported the use of glass beads. Following a cleaning step, the glass beads were handled and rolled in the palms of the volunteers for $10 \mathrm{~min}$. Hexane was then used to extract the scent molecules [58].

As for breath analysis, such a straightforward and noninvasive in vivo skin sampling holds high interest and potential in the medical field. However, so far, not many reports have made use of this alternative in vivo sampling techniques with $\mathrm{GC} \times \mathrm{GC}$, and further studies are required to understand their full capabilities.

\subsection{3 | In vitro and cell culture-based}

In vitro research is of particular interest since it enables the study of biological processes leading to the production of VOC and SVOC in an "easy-to-manipulate" environment compared to in vivo systems.

HS-SPME is a commonly used approach in in vitro studies, mainly due to its simplicity, reproducibility, and possible automation [41,59-65]. Recently, this approach was employed to study the volatile metabolites from lung epithelial cells subjected to chemical and biological inflammation using, hydrogen peroxide and sputum from asthmatic patients, respectively [65]. A DVB/CAR/PDMS fiber was selected to extract the widest range of VOCs emitted from the cell culture subjected to the different stressors. According to the type of inflammation induced and the associated status of the cells, different volatile metabolites have been characterized. Increased production of volatile compounds was reported following the co-incubation of epithelial cells with inflammatory sputum when compared to cells treated with non-inflammatory sputum and non-treated cells. The principal component analysis of Figure 6 is demonstrating the differentiation between chemically and biologically stressed epithelial cells and three different cellular controls consisting of non-treated cells (green), cells diluted in PBS that is used to treat the sputum samples (yellow), and cells treated with sputum from healthy volunteers (pink). Such clear differentiation is confirming the potential of volatile metabolites to reflect the status of lung epithelial cells and therefore, alterations in their metabolism.

HS-SPME has also been used to characterize virally infected respiratory cells [60] and to study the volatile profile of numerous bacteria and fungi $[66,67]$. Martins et al. have evidenced differences in the extracellular volatile metabolites of two different yeasts found in beer and wine [66]. The analysis, using HS-SPME, of $S$. cerevisiae and 


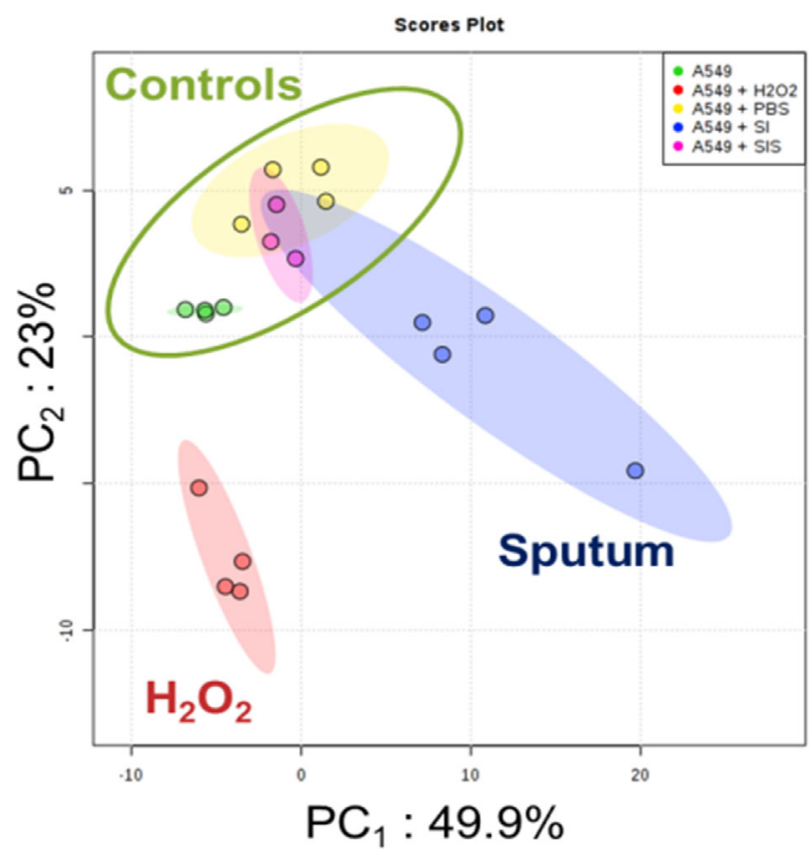

F I G U RE 6 Principal component analysis of the chemical and biological induced inflammation on A549 lung epithelial cells using selected features. Adapted and reproduced from [65], with permission from the Royal Society of Chemistry

S. pastorianus, at different sampling points corresponding to different growth phases (lag, exponential, and logarithmic), revealed a high number of compounds that were correlated with specific metabolic pathways. A similar approach was used to study the extracellular and intracellular metabolites on bacteria exposed to heavy metals [67]. In this case, the rapid extraction of bacterial volatiles (the extracellular and intracellular fractions were obtained in only $15 \mathrm{~min}$ and a $60 \mathrm{~min}$ SPME fiber exposure was necessary to extract the volatile compounds) allowed identifying the evolution in the volatile metabolome to further understand bacterial survival in a contaminated environment. Mousavi et al. reported the use of HS-SPME to successfully follow metabolic changes of $E$. coli induced by natural antibacterial agents such as clove oil and eugenol in realtime and in vivo [68]. The treatment of $E$. coli with clove oil induced a dysregulation of 125 volatile metabolites, among which derivatives formed along with the fatty acid biosynthetic pathways, such as alcohols, ketones, and esters, were the most affected. This exposition of SPME fiber during the bacterial growth (in vivo) enhanced the amount of chemical information that can be obtained from the biological system under study while avoiding any a priori sample manipulation. Similarly, an HSSE method has been exploited to characterize antifungal VOC activity of bacteria and enabled to highlight new volatile metabolome properties from the co-culture of bacteria and fungi [69].
Thermal desorption tubes also represent a valid alternative for in vitro research sampling. The need for consistent analyte extraction between in vitro and in vivo studies for biomarker discovery and translation (e.g., breath or urine and cell cultures) has been recently reported [42]. The authors highlighted the importance of the sorbent material selection for the trap tubes in bacterial VOC sampling from cultures. Indeed, the physicochemical characteristics of the numerous adsorbent materials available are influenced differently by the sampling conditions (e.g., humidity) and sample complexity, affecting the final results. Figure 7 shows the heatmap of three bacterial strains (P. aeruginosa, $S$. aureus, E. coli) involved in lung infections and obtained with two differently packed traps. The better separation of the three bacterial strains was highlighted when using Tenax TA, thanks to its higher reproducibility and metabolites coverage during the sampling.

Despite the advantages of HS-based sampling, the study of cell and bacteria metabolism often requires the characterization of SVOCs, which are intrinsically more interrelated with the matrix and can be more challenging to extract. In these cases, conventional extractions followed by derivatization techniques are the approaches generally used. As an example, Miguez et al. used silylation agents to study small polar metabolites from the lycopene and mevalonate pathways of E. coli [70]. Among these, homocysteine and homoserine have been identified as possible growth inhibitors associated with the overnight induction of the mevalonate pathway of E. coli. Their optimized quenching protocol allowed the measurement of the metabolites over $6 \mathrm{~h}$ with a high turnover rate. The protocol provided an increased understanding of the central carbon metabolism of $E$. coli that can be used to drive pathway and strain engineering.

\subsection{Food- and plant-related}

The non-targeted analysis of metabolites in food- or plant-related products commonly aims for obtaining a chemical snapshot of the sample composition. Regarding food-related products, the detection of small molecules such as acids, alcohols, aldehydes, carbonyl compounds, esters, ketones, phenols, or terpenoids is of high importance as this information can be used for a variety of applications. For example, it allows controlling food production processes, maintaining or improving food quality, or developing a particular organoleptic property. In plants instead, the chemical signature is related to a particular metabolic setting of a physiological/altered state. The study of small metabolites as a picture of the metabolic state using high-resolution techniques appeared in early 2000 and progressed until nowadays 

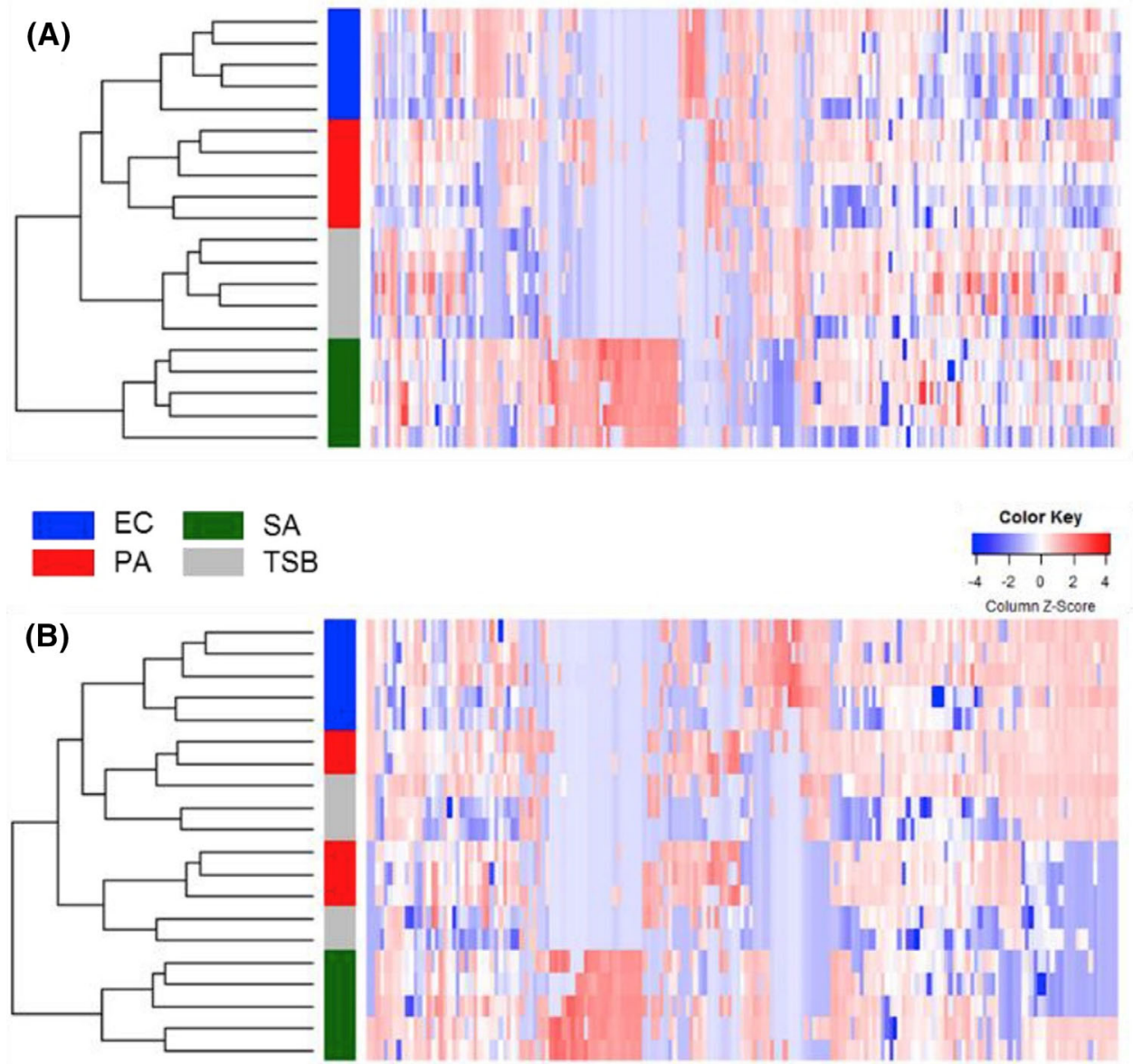

F I G U R E 7 Heatmap and hierarchical cluster analysis showing the clustering of the control, and SA, PA and EC cultures using (A) Tenax TA and (B) CarbopackY/CarbopackX/Carboxen1000 as sorbent materials. Adapted and reproduced from [42] with permission from Elsevier

with the continuous implementation of cutting-edge analytical technologies [71]. In the context of GC $\times \mathrm{GC}$, the recent years have seen the majority of the non-targeted analysis in food and plant-related samples focusing on the more volatile metabolites, thus exploiting HS techniques (94\%) involving GPe (DHS, purge-and-trap (P\&T), static headspace (SHS)), and SPe (HS-SPME, HSSE).

Especially miniaturized sampling techniques allowing for pre-concentration such as SPME, HSSE, and DHS have shown to be useful to limit artifact formation and match the analytical requirements of automation [72]. It has become more and more evident that the hyphenation of a robust sample preparation technique with advanced GC $\times$ GC separation and MS detection is important and that suitable software and data-handling tools with statistical analysis are necessary to make proper use of the highdensity acquired information.

Regarding food- and plant-related products, $82 \%$ of the revised literature of the last 5 years used non-targeted approaches (metabolite profiling and/or fingerprinting), $18 \%$ aimed for a targeted approach, and $~ 80 \%$ involved the use of HS-SPME.

\subsection{1 | Beverages}

The understanding of the aroma composition, for example, to improve sensory properties, is one of the main focus in the analysis of beverages. The aroma, which can be considered as a major consideration for consumers' choice, derives primarily from its ingredients; however, it is also strongly influenced by its production process. Concerning the aroma and sensory studies of any kind of beverage, surely the headspace sampling is the primary and most used technique exploited with $\mathrm{GC} \times \mathrm{GC}$ [72].

The majority of the revised literature investigating alcoholic beverages used SPME in HS-mode for aroma analysis. HS-SPME in combination with FM-GC $\times$ GC-MS has been used to distinguish beers fermented with two different yeast strains. Multiway principal components analysis revealed 46 compounds, among the approximately 210 detected, which were relevant for the classification [73]. Paiva et al. chose the commercially available DVB/CAR/PDMS fiber to obtain the widest analyte coverage for their non-targeted analysis. 
Static (classical SHS, HS-SPME) and dynamic HS extraction techniques (DHS) have been compared for the aroma analysis of Belgian Trappist beers [74]. The authors also tested the extraction efficiency of classical and multiple SBSE, concluding that DHS exhibited a satisfying trapping efficiency for aroma VOCs while producing limited chromatographic artifacts. An alternative dynamic sampling, involving the use of P\&T (Tenax TA) was explored with a FM-GC $\times$ GC-MS system, discussing a straightforward and informative workflow for aroma analysis on different fruity beers [75]. As for the DHS, purge-and-trap represents a long-established dynamic technique, which consists of pushing the VOCs continuously from the liquid sample (bubbling an inert gas through the sample) into the HS, resulting in a more efficient extraction of the analytes. Also, this study is the first published paper reporting on the combination of purge-and-trap with GC $\times \mathrm{GC}$.

In the quest for unique selectivity, ionic-liquid coatings have been recently developed for sampling methods $[76,77]$, in addition to their use as stationary phase for GC columns [78,79]. Few recent pieces of research reported on the use of poly ionic-liquid (PIL)-based sorbent coatings for HS-SPME in $\mathrm{GC} \times \mathrm{GC}[80,81]$. Crucello et al. investigated several non-ionic and PIL-based sorbent coatings for HS-SPME, analyzing VOCs in Brazilian wine [80]. About 350 peaks were detected in four wine samples. Among the five different fibers tested, the DVB/CAR/PDMS and one of the PIL coated fibers exhibited improved selectivity toward a broad range of aroma VOCs present in wine. That particular PIL-coated fiber extracted more effectively polar analytes found in wine compared to the popular DVB/CAR/PDMS fiber because significantly higher distribution coefficients can be obtained with ionic liquid coatings. Noteworthy is that some compounds were solely detected employing the aforementioned PIL fiber. For example, two of the four potential markers to distinguish "Isabella" and "BRS Magna" wine cultivars, namely ethyl butanoate and ethyl methyl succinate, were only extracted when using the PIL-based SPME fiber.

Similarly, for non-alcoholic beverages such as tea and coffee, HS-SPME has been extensively applied for the analysis of infusions or dried plant material [82-84]. Nevertheless, also the direct immersion approach (DI-SPME) [82], simultaneous distillation extraction (SDE) [85], and derivatization [86] have been reported.

Aroma components of several green tea and chestnut were analyzed by Zhu et al., using two different sample preparation techniques (SDE and HS-SPME) [87]. The authors considered SDE as the most suitable sample preparation technique for green tea aroma; however, due to the poor solubility and volatility of chestnut aroma compounds in the SDE solvents, HS-SPME was applied as an alternative extraction technique. Eventually, (1) the com- bination of SDE with $\mathrm{GC} \times \mathrm{GC}-\mathrm{MS}$ used to determine the odor activity value, and (2) the HS-SPME/GC-MS/GC-O used for the identification of key odorants, revealed eight VOCs as the most definite odorants to promote the formation of the chestnut-like aroma of green tea.

Magagna et al. sampled VOCs from the HS of dried black tea leaves in different conditions (i.e., untreated, suspended, and infused in water) [82]. The dry plant material was sampled via HS-SPME, SBSE, and DHS and the infusion via DI-SPME, and SBSE. The efficiency of each sampling method was compared in terms of numbers of analytes extracted and their response. Significant differences in extraction capabilities were shown, depending on the technique used. On one hand, DHS and HSSE (both using PDMS) revealed good recovery of less polar and low volatility analytes such as saturated aldehydes $\left(\mathrm{C}_{10}\right.$ to $\left.\mathrm{C}_{18}\right)$, methyl ketones $\left(\mathrm{C}_{11}\right.$ to $\left.\mathrm{C}_{15}\right)$, and some medium-chain alcohols. On the other hand, HS-SPME (DVB/CAR/PDMS) showed the highest suitability for highly-volatile aldehydes $\left(\mathrm{C}_{5}\right.$ to $\left.\mathrm{C}_{7}\right)$ and was therefore considered by the authors as the most suitable sampling technique to establish aroma fingerprinting. Nevertheless, the authors suggested that the most comprehensive chemical signature would be provided by the combination of the complementary information offered by the different sampling approaches.

Similarly, the chemical composition of raw coffee has been correlated to the end products' quality, authenticity, and sensory properties [88]. Novaes et al. reported a method for the analysis of low volatility compounds in green Arabica coffee extracts without the need for prior fractionation or another pre-treatment (e.g., derivatization) [89]. After liquid-liquid extraction (at circa $77^{\circ} \mathrm{C}$ ), samples were subjected to high-temperature GC and GC $\times$ GC. The GC $\times$ GC set-up, consisting of a relatively short $1 \mathrm{D}$ column $(11 \mathrm{~m})$ and therefore requiring the optimization of some separation conditions such as trap temperature, oven temperature program, and flow rate, allowed for the elution also of thermo-labile and low volatility compounds. Despite minimal sample preparation, superior performances of $\mathrm{GC} \times \mathrm{GC}$ in comparison to GC resulted in improved compound identification and group-type analysis of the approximately 200 detected compounds. The same authors developed a sampling protocol for the trapping of VOCs and SVOCs during the roasting process of coffee beans [88]. For the dynamic sampling, the authors tested two different sorbents (XAD-2 and CSC). Figure 8 displays the $\mathrm{GC} \times \mathrm{GC}$ results for VOCs and SVOCs extraction of the coffee roasting process using XAD-2 (left side) and CSC (right side) adsorbents for different time-intervals. The boxed zones highlight SVOCs elution regions. Figure 8 readily demonstrates that the use of XAD-2 for the sample preparation, in combination with a high-temperature column set for the two-dimensional 


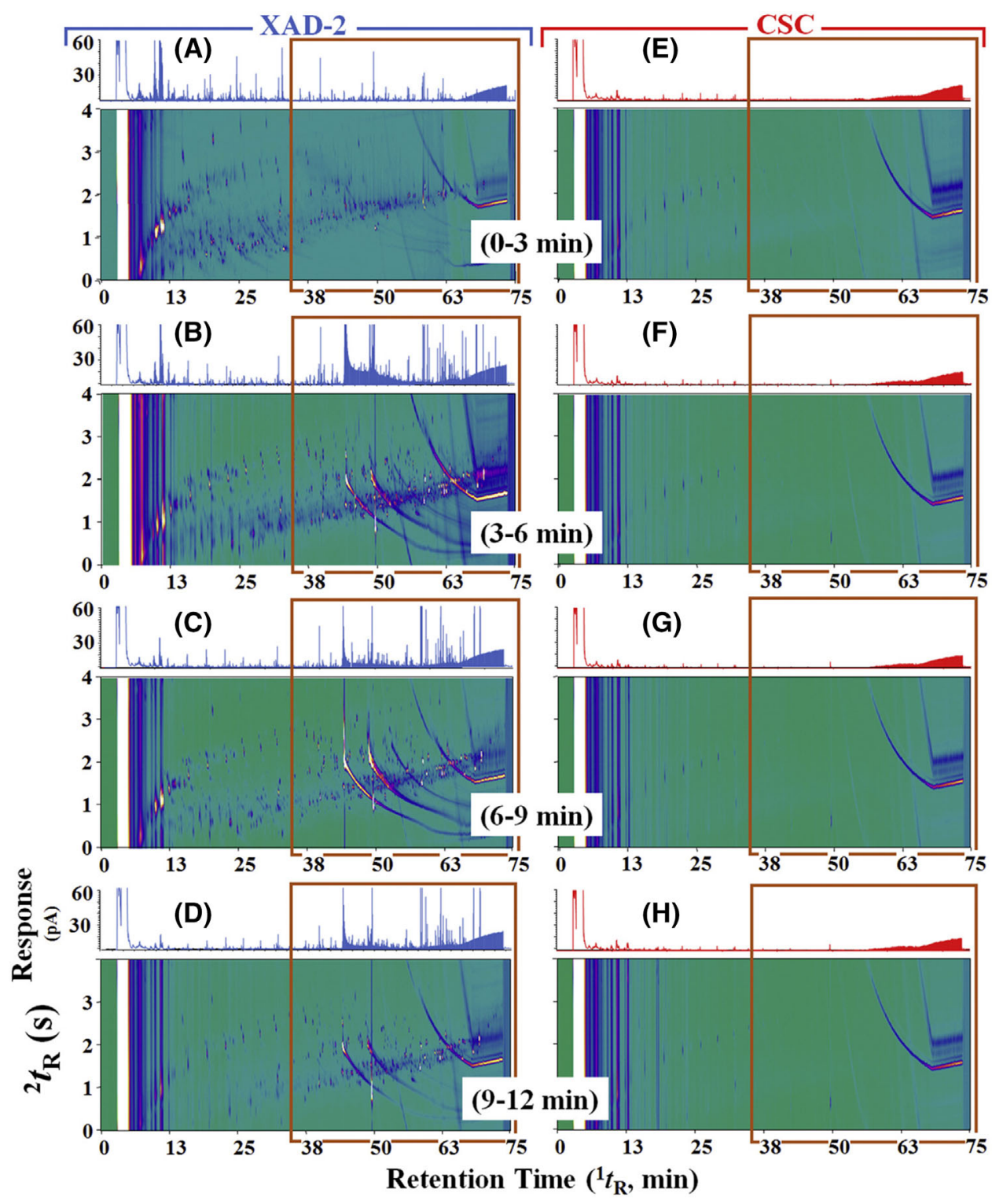

F I G U R E 8 GC $\times$ GC results for VOCs and SVOCs extraction (DHS) of the coffee roasting process using flow-through/active sampling with two different adsorbents (XAD-2 and CSC, on the right and on the left, respectively) at different roasting time intervals. The boxed zones highlight SVOC elution regions. Adapted and reproduced from [88] with permission from Elsevier

separation allowed the specific identification of SVOCs for the chemical profiling of the roasting process.

\subsection{2 | Plant-based edibles}

The assessment of quality represents often a central aim in the research field of edibles, and the combination of robust sample preparation and high-resolution separation techniques contribute to provide dense information about sample composition. Among the most common food products derived from plants, cocoa and hazelnut composition have been studied deeply using HS techniques [90-94]. For example, the volatile metabolome of cocoa from dif- ferent regions was analyzed using HS-SPME and GC $\times$ GCMS [90]. The SPME method was standardized, pre-loading an internal standard ( $\alpha$-thujone) onto the fiber before sampling for the normalization of analyte response. In their study, they exploited the complexity of the cocoa headspace to develop new workflows for non-targeted and targeted analysis. Also, they highlighted the value of the combination of softer ionization with conventional electronic ionization to increase the level of confidence for the identification of VOCs.

The HS of vegetable oils generally carries sensoryrelevant information which can be used not only to define the quality but also to discriminate different varieties and geographical origins. HS-SPME was used by Lukić et al. for 


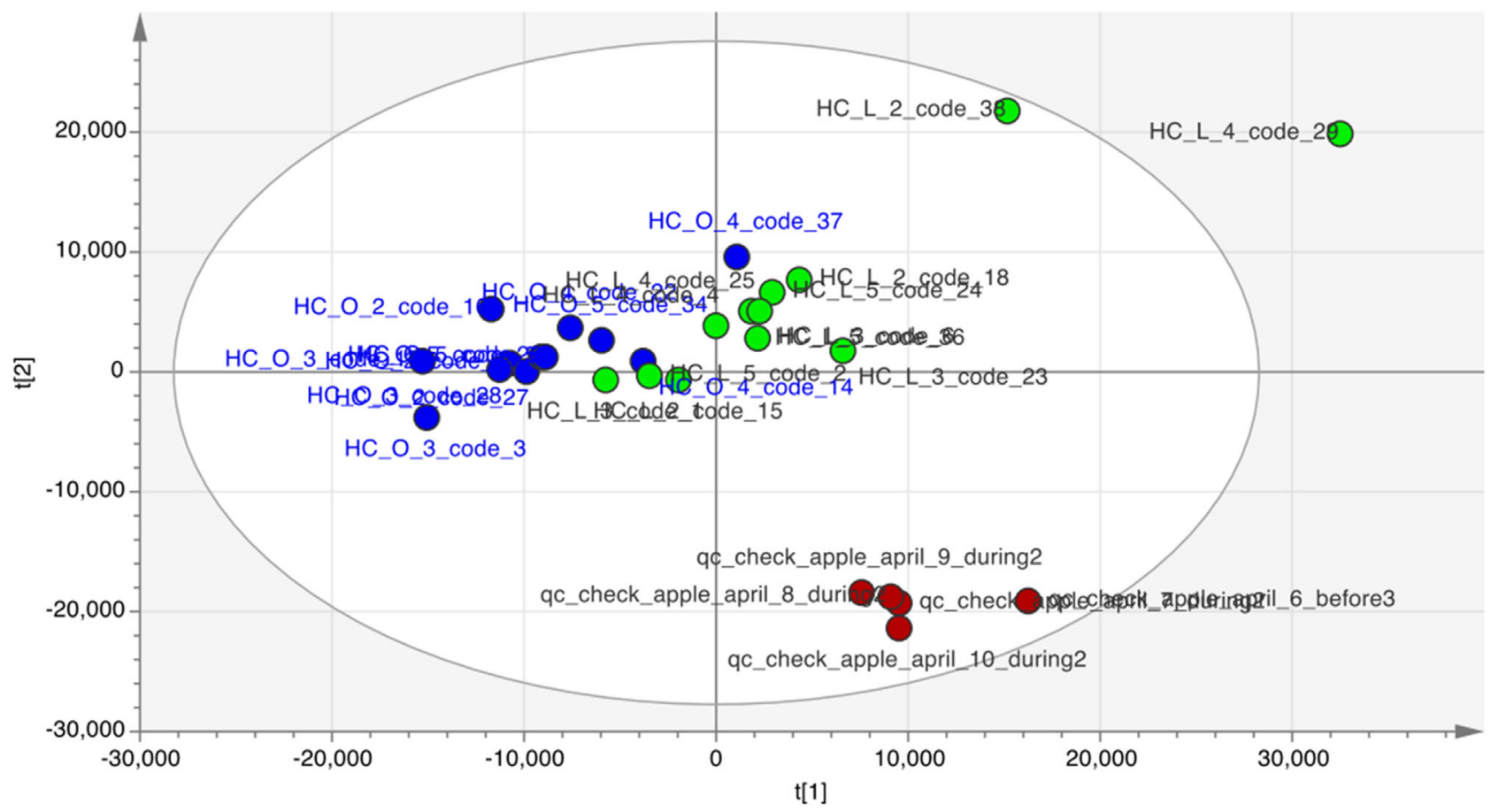

F I G U R E 9 PCA scores plot of in vivo DI-SPME data for apples with higher maturity index (green circles), apples with lower maturity index (blue circles), and ex vivo HS-SPME data obtained for control samples (red circles). Reproduced from [100]

the characterization and differentiation of virgin olive oils made from different varieties grown in different regions [95].

Multiple headspace sampling techniques have been applied to study the complex volatilome of olive oils [96]. The extraction capabilities of static (HSSE, HSSPME), monolithic material sorptive extraction, and dynamic headspace extraction (DHS), were compared as well as several sorbent materials (DVB/CAR/PDMS, PDMS, PDMS/Carbopack B, PDMS/EG, ODS, ODS/GC, and Tenax TA). Interestingly, the authors pointed out that the sampling techniques with higher amounts of sorbent, such as HSSE and DHS (Tenax TA), gave better results in terms of concentration capacity, resulting in a higher extraction yield. However, they focused the attention principally on the use of the HS-SPME approach and discussed how the combination of such a technique can successfully be integrated with GCXGC-MS.

On the less volatile fraction of vegetable oils, derivatization approaches for the characterization of the minor components (unsaponifiable fraction) were also proposed, combining a silylation method with GC $\times$ GC-high resolution (HR) MS [97] and a miniaturized-SPE with dual detection GC $\times$ GC-MS/FID [98].

After the relevant contribution from Risticevic et al. showing the potential of DI-SPME [16], further in vivo and in situ apple metabolome studies have been performed $[99,100]$. In these studies, the authors confirmed the fea- sibility of the approach to provide unbiased metabolite coverage, compared to traditional approaches requiring metabolism quenching and laborious sample preparation. Apple was used as a living plant sample to detect changes in metabolic fingerprints in response to fruit maturation. An overcoated triphasic SPME fiber was used, showing more effective cleanup and reduced decomposition products. In these works, esters were the most important contributors to the aroma profile of apples, both in quantitative and qualitative terms. Also, the comprehensive metabolome coverage obtained with in vivo DI-SPME was reflected by the extraction of aldehydes, ketones, alcohols, aromatic compounds, carboxylic acids, aromatic aldehydes, aromatic ketones, benzyl alcohols, alkanes, benzyl acetates, and glycol ethers, as well as metabolites originating from the lipoxygenase pathway. For the esters profiling, the structurally ordered $\mathrm{GC} \times \mathrm{GC}$ chromatograms supported the identification of 13 acetates, 5 propanoates, 10 butanoates, and ethyl hexanoates. Interestingly, a comparison of the in vivo sampling on the intact apple with the ex vivo sampling following homogenization and metabolism quenching, revealed differences in metabolites, corroborating the fact that the metabolome is influenced by the sample preparation procedure and that many metabolites can be produced or altered during this step (Figure 9).

The analysis of fatty acids in foodstuff is another common area of research, which typically involves the derivatization and isolation of targeted classes before the $\mathrm{GC} \times \mathrm{GC}$ 

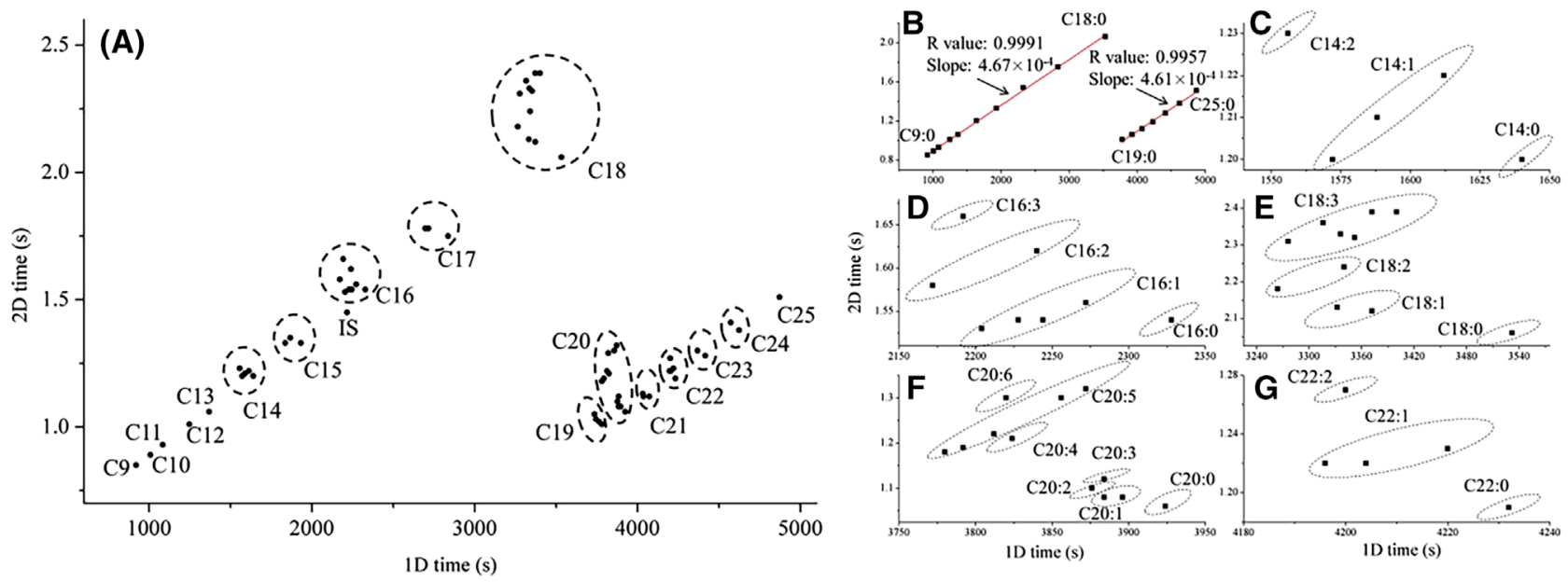

F I G U R E 10 Apex/dot plot with the identified FFAs in edible oils using the MDE followed by GC $\times$ GC-MS (a). Saturated FFAs (b), $\mathrm{C}_{14: \mathrm{N}}$ (c), $C_{16: N}(d), C_{18: N}(e), C_{20: N}(f), C_{22: N}(g)$, in edible oils using the magnetic dispersive extraction followed by GC $\times$ GC-MS analysis. $C_{\mathrm{x}: \mathrm{y}}$ represent the carbon number and the unsaturation degree of the fatty acids. Reproduced from [101] with permission from Elsevier

separation. The determination of free fatty acids in edible oils is particularly important for quality and authenticity control, and it was recently investigated by performing magnetic dispersive extraction (MDE) followed by derivatization using silylation agents [101]. The extraction method combined the advantages of magnetic solidphase extraction and dispersive liquid-liquid microextraction. However, the multiple steps and the emulsification issues can impede its widespread use. After the extraction, free fatty acids were derivatized using silylation agents, concentrated, and then injected in a GCXGC-MS system. The authors highlighted the help given by the regular and predictable patterns of the homolog fatty acids, which facilitated the qualitative analysis of fatty acids without the use of analytical standards (Figure 10).

\subsection{3 | Plant parts and extracts}

The non-targeted analysis of plant parts and extracts usually aims at the better characterization of the samples and understanding of biochemical processes, and the acquired knowledge could support in correlating the health-beneficial properties of the natural products.

The composition of a variety of plant extracts or resins used in traditional oriental medicine has been characterized by GCXGC $[102,103]$. The analysis of these typically complex natural products benefits substantially from the increased separation power allowing for the precise determination of the compounds with potential biological activity [102]. In a comparative study of the volatile metabolite profiles of resin from five Dracaena species, HSSPME-GC $\times$ GC-MS allowed identifying 20 terpenoid components. It was found that the terpenoid compounds are species-specific and that the monoterpene fraction could serve to determine chemotaxonomic markers [103].

The VOCs from Frankincense (the gum resin of Boswellia papyrifera), which also present potent antimicrobial activities, were studied using a PDMS/DVB SPME fiber allowing the detection of about 220 compounds in a single analysis [104]. Group-type separation allowed for the classification of monoterpenes, sesquiterpenes, and diterpenes, and unidentified compounds could be further classified based on the elution pattern and mass spectral information.

A continuously emerging field is the analysis of cannabis and its related products, which are mainly used for their psychoactive and medicinal activities. About 500 compounds have been identified and, among them, cannabinoids and terpenoids currently hold the highest interest for their biological and synergistic activity. Conventional studies have led to protocols optimized for the analysis of a few targeted analytes or chemical classes. Instead, GCXGC has shown its advantages in the multiclass and high-resolution analysis of a variety of analytes $[105,106]$. In a recent approach, a methodology involving the use of SBSE was employed for the multiclass metabolite profiling of cannabis inflorescences [107]. The SBSE was selected to cover the high molecular weight metabolites, and particular attention was dedicated to the optimization of the extraction conditions in order to extend the analytes class coverage (Figure 11). Using a fractional factorial design, five chemical classes were considered to optimize the extraction conditions in a non-targeted fashion. Figure 11A shows the response trends under the different extraction variables and levels considering the investigated classes, which were group-type separated in the $\mathrm{GC} \times \mathrm{GC}$ space and tracked using characteristics 

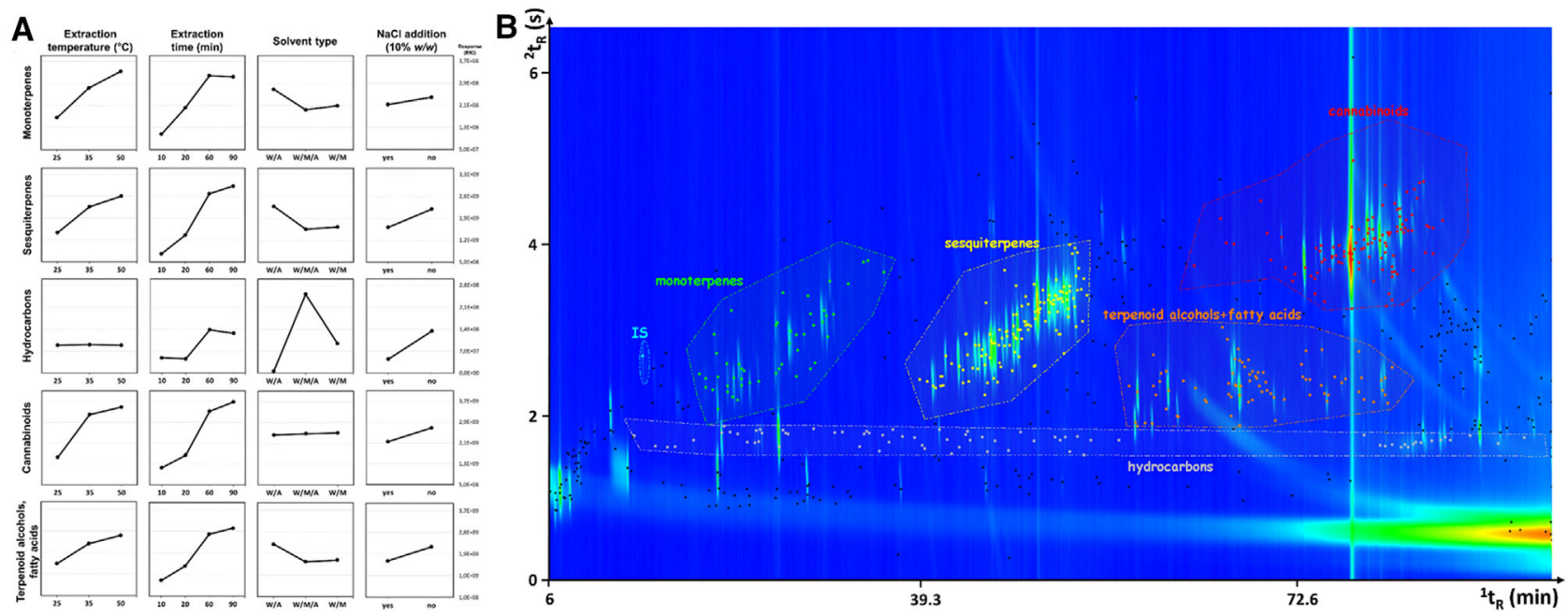

F I G U R E 11 A) Response plots under different extraction conditions of monoterpenes, sesquiterpenes, hydrocarbons, cannabinoids, and terpenoid alcohols and fatty acids. B) SBSE FM-GC $\times$ GC-MS chromatogram of a cannabis inflorescence sample. Adapted and reprinted with permission from [107]. Copyright 2020 American Chemical Society

ions (Figure 11B). Under optimized conditions, different cannabis chemovars belonging to sativa, indica, and hybrid subspecies were analyzed combining SBSE with a FM-GC $\times$ GC-MS system. In addition to chemotype classification, quantitative analysis was performed on three common cannabinoids (THC, CBD, and CBN). Noteworthy, the non-targeted sampling strategy found also confirmation with the detection of exogenous compounds like pesticides, plasticizers, and cannabinoids degradation products, which were identified with high confidence by using high-resolution MS (SBSE-GC $\times$ GC-HR MS).

Some high molecular weight molecules, such as small carbohydrates and phenylalkanoid glycosides play an important role in the biological activity of plants. Even though these molecules are typically analyzed via HPLC, Carrero-Carralero et al. reported a derivatization protocol employed in combination with $\mathrm{GC} \times \mathrm{GC}$ for the analysis of Sedum roseum root dietary supplement [108]. In this uncommon choice of analyzing sugar and glycosides, the authors highlighted the importance of the optimization of the derivatization step (silylation) for the class of phenylpropanoid glycosides. The final derivatization procedure allowed for the complete and reproducible identification and quantification of targeted compounds with a GC-based system. Also, the non-targeted analysis revealed the presence of a variety of other important compounds such as polyphenols, terpenes, phenylalkanoids, carbohydrates, and glycosides which were co-extracted and separated in the high-resolution chromatographic system.

Essential oils and other plant extracts from aromatic plants are widely appreciated in the food and flavor industry due to their olfactory properties. Essential oils, typically obtained by hydrodistillation, are mainly injected directly, although a sample preparation step beforehand can be ben- eficial to increase selectivity. For example, an additional liquid-liquid extraction combined with high-resolution GC $\times$ GC-MS (QTOF) was used for the detailed chemotypic profiling of essential oils of different hop genotypes [109]. Bendif et al. compared the extract of T. munbyanus obtained via hydrodistillation, pressurized liquid extraction (PLE), and supercritical fluid extraction (SFE) [110]. The authors discussed the impact of solvent polarity using PLE, and how the different ratios resulted in various total phenolic content which was related to the antioxidant activities. However, major differences were observed in the metabolic profile of essential oils obtained via hydrodistillation, mostly characterized by the presence of typical terpenoids, whereas the more lipophilic SFE extracts contained higher amounts of long-chain hydrocarbons and tocopherols.

Other pre-separation procedures, especially those based on chromatographic techniques have been proposed to select specific fractions of interest. Tissandie et al. reported a detailed investigation of vetiveryl acetates and vetiver oils and related it to the final olfactory properties [111]. A series of SPE cartridges (i.e., silica and silver) were used to isolate the different chemical classes before $\mathrm{GC} \times \mathrm{GC}$ MS/FID for qualitative and quantitative analysis. The authors exploited the different SPE characteristics and elution solvents polarity to obtain a selective fractioning of the samples (i.e., hydrocarbons, esters, aldehydes and ketones, tertiary alcohols, and vetivones) for detailed characterization. The authors finally concluded that the $\mathrm{GC} \times \mathrm{GC}$ analysis of vetiveryl acetates allowed more reliable quantification of their constituents, superior to what the conventional GC-FID could offer.

Another powerful approach that also holds the possibility for automation and on-line configuration, is the 
use of liquid chromatography as a pre-fractionation step before the GC $\times \mathrm{GC}$ separation. A detailed qualitative analysis of the volatile fractions of mandarin and lime essential oils was achieved by combining HPLC and GC $\times$ GCMS [112,113], although in an off-line configuration in these examples. The pre-separation using a normal phase HPLC process aimed at the isolation of the oxygenated compounds contributing to the composition of the essential oils.

Hydrodistillation followed by a hybrid heart-cut GC-GC and GCXGC-MS system was combined for the analysis of hop (Humulus lupulus L.) essential oil and agarwood (Aquilaria malaccensis) oleoresin [114]. This represents a neat example to show the close relation of sample preparation and multidimensional chromatography in contributing to the removal of interferences and the increase of sensitivity. In this configuration, the initial heart-cut GC-GC acted as the sample preparation step and replaced a conventional fractionation step, allowing to isolate the fraction of interest which was then further subjected to the high-resolution GC $\times$ GC separation. Figure 12 shows the analysis of oxygenated sesquiterpenes in agarwood oleoresin using GC-FID (Figure 12A), heart-cut GC-GC (Figure 12B), and GC-GC $\times$ GC-MS (Figure 12C). An extension of compound coverage concerning oxygenated sesquiterpenes was apparent when samples were subjected to the high-dimensional separation system (GC-GCXGC-MS).

Generally, the use of DI-SPME appears not common, especially in the case of matrices with high-fat content, and due to issues related to the direct exposure of the coating with the matrix (lack of reproducibility, poor sensitivity, or insufficient ruggedness).

Noteworthy, DeGrazia et al. [115] presented an approach for DI-SPME analysis of high-fat content matrices (in avocado puree). A commercially available PDMS/DVB fiber was over-coated with an additional layer of PDMS to enhance inertness and prevent fouling of the fiber surface. Critical parameters for the sample extraction such as pre-desorption rinsing and post-desorption washing were optimized for time, solvent mixture, and agitation conditions, to assure satisfactory sensitivity and reproducibility of the method [4]. In their study, the utilization of GC $\times$ GC provided sufficient resolution for the evaluation of the fiber coatings. Indeed, the multidimensional separation allowed the assessment of the degree of matrix accumulation in the fiber and/or the formation of artifacts at the injector port, for example, resulting in high background signals and very complex 2D chromatograms. The modified PDMS/DVB/PDMS coating showed superior performance regarding its robustness which permitted more than 100 consecutive extraction cycles. Finally, the method was automated, resulting in a high-throughput approach with minimal sample preparation for food analysis.
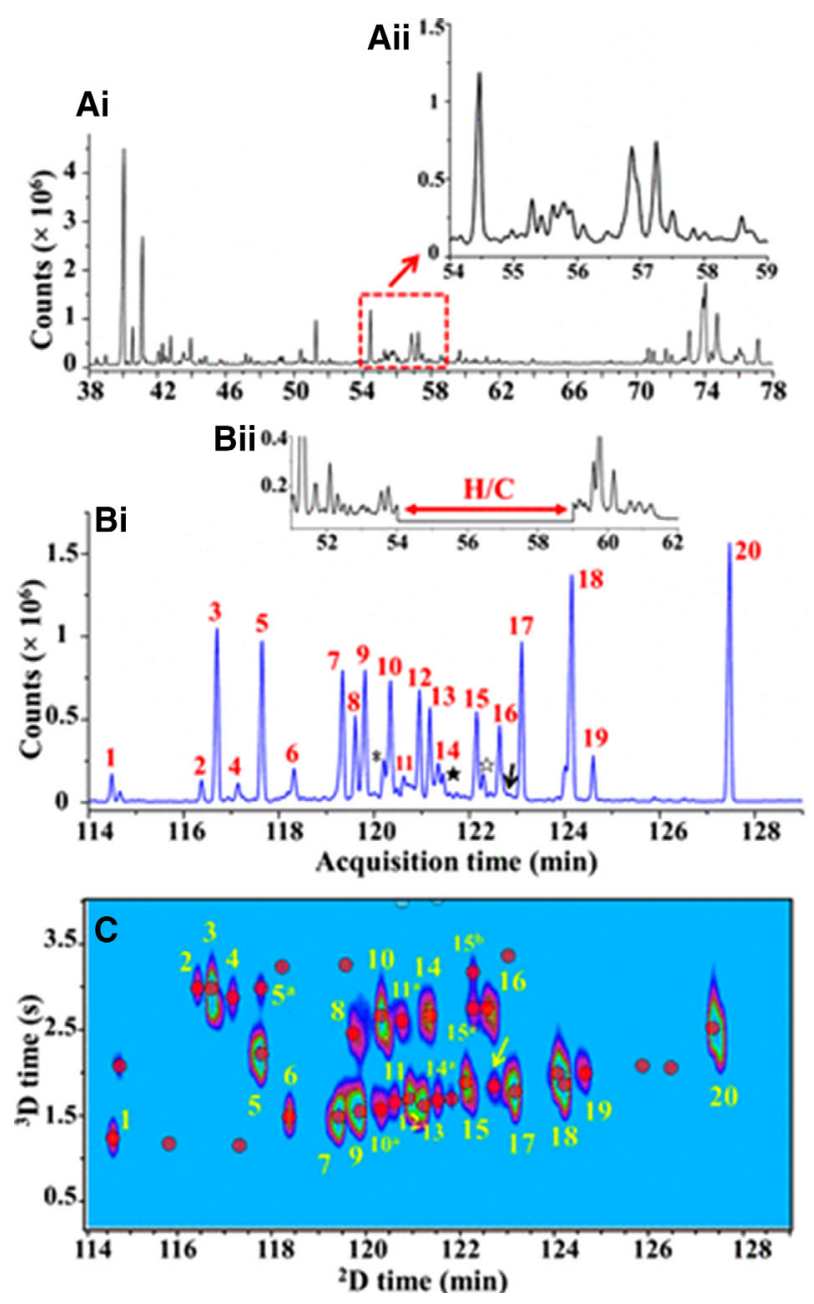

F I G U R E 12 Sequential GC-GC $\times$ GC-MS analysis of the oxygenated sesquiterpenes in $A$. malaccensis oleoresin. ( $\left.\mathrm{A}_{\mathrm{i}}\right)$ 1D FID response, the region to be heart-cut $(\mathrm{H} / \mathrm{C})$ is denoted by the dotted rectangle, with an inset $\left(\mathrm{A}_{\mathrm{ii}}\right)$ of the expansion of the target region. $\left(B_{i}\right)$ H/C GC-GC-MS analysis of the target region and with an inset $\left(B_{i i}\right)$ of the 1D FID response. (C) GC-GC $\times$ GC-MS analysis of the target region. Reprinted with permission from [114]. Copyright 2020 American Chemical Society

\section{4 | CONCLUSIONS AND PERSPECTIVES}

The affinity and complementarity between a generic sample preparation technique and the $\mathrm{GC} \times \mathrm{GC}$ separation was herein considered and commented. Recent biomedical, food, and plant applications (2016-2020) were also discussed, specifically those in which non-targeted analysis was sought, and the combination of tailored sample preparation methods and GCXGC-MS has proven to be beneficial.

Even though common features between a typical sample preparation and the $\mathrm{GC} \times \mathrm{GC}$ separation can be identified (Figure 1), the full optimization of the two processes remains fundamental for reliable and reproducible results, especially in the challenging aspects of non-targeted 
analysis in biomedical, food, and plant applications. Moreover, their combination with the identification capability of mass spectrometry generates the most powerful and flexible analytical platform for complex sample analyses. Indeed, a complete analytical methodology that exploits (1) a wide and an unbiased analyte extraction from the sampling step, and (2) the sensitivity, the separation, and identification power from the $\mathrm{GC} \times \mathrm{GC}$ separation and the MS detection, represents a solid advantage for non-targeted analysis.

In the quest for greener, miniaturized, faster, selective/universal, and robust sampling strategies, it will be interesting to see how some recently developed sampling alternatives (vacuum-based, thin-film, paper-based, freeze-concentration, or solvent-assisted SPe techniques [116-119]) will perform with GC $\times$ GC.

An additional aspect to consider indeed is the use of $\mathrm{GC} \times \mathrm{GC}$ to discover new sample preparation techniques and to fully exploit, evaluate, and refine the existing ones. In fact, the $\mathrm{GC} \times \mathrm{GC}$ increased separation power provides a more comprehensive overview of the sampling performance, and finally of the sample composition.

\section{ACKNOWLEDGEMENTS}

This article is based upon work from the Sample Preparation Task Force and Network, supported by the Division of Analytical Chemistry of the European Chemical Society. Flavio A. Franchina was funded by the FWO/FNRS Belgium EOS grant 30897864 "Chemical Information Mining in a Complex World." Delphine Zanella was funded by the Fund for Industry and Agricultural Research (FRIA).

\section{CONFLICT OF INTEREST}

The authors have declared no conflict of interest.

\section{ORCID}

Flavio A. Franchina (D) https://orcid.org/0000-0001-72364266

Delphine Zanella (1) https://orcid.org/0000-0002-0131-

2837

Lena M. Dubois (1) https://orcid.org/0000-0002-0084-0320 Jean-François Focant @ https://orcid.org/0000-0001-80752920

\section{RE F E RE N C ES}

1. Liu Z, Phillips JB. Comprehensive two-dimensional gas chromatography using an on-column thermal modulator interface. J. Chromatogr. Sci. 1991;29:227-31.

2. Phillips JB, Beens J. Comprehensive two-dimensional gas chromatography: A hyphenated method with strong coupling between the two dimensions. J. Chromatogr. A. 1999;856:33147.
3. Tranchida PQ, Maimone M, Purcaro G, Dugo P, Mondello L. The penetration of green sample-preparation techniques in comprehensive two-dimensional gas chromatography. TrAC Trends Anal. Chem. 2015;71:74-84.

4. Pawliszyn J, Heather LL. Handbook of Sample Preparation. Edited by Pawliszyn J, LL Heather. Hoboken, N.J: Wiley, 2011.

5. de Souza JRB, Dias FFG, Caliman JD, Augusto F, Hantao LW. Opportunities for green microextractions in comprehensive two-dimensional gas chromatography / mass spectrometrybased metabolomics - A review. Anal. Chim. Acta. 2018;1040:118.

6. Bojko B, Reyes-Garcés N, Bessonneau V, Goryński K, Mousavi F, Souza Silva EA, Pawliszyn J. Solid-phase microextraction in metabolomics. TrAC - Trends Anal. Chem. 2014;61:168-80.

7. Arthur CL, Pawliszyn J. Solid phase microextraction with thermal desorption using fused silica optical fibers. Anal. Chem. 1990;62:2145-8.

8. Adahchour M, Beens J, Vreuls RJJ, Batenburg AM, Rosing EAE, Brinkman UAT. Application of solid-phase micro-extraction and comprehensive two-dimensional gas chromatography (GC x GC) for flavour analysis. Chromatographia. 2002;55:361-7.

9. Perera RMM, Marriott PJ, Galbally IE. Headspace solid-phase microextraction - Comprehensive two-dimensional gas chromatography of wound induced plant volatile organic compound emissions. Analyst. 2002;127:1601-7.

10. Gaines RB, Ledford EB, Stuart JD. Analysis of water samples for trace levels of oxygenate and aromatic compounds using headspace solid-phase microextraction and comprehensive two-dimensional gas chromatography. J. Microcolumn Sep. 1998;10:597-604.

11. Kueh AJ, Marriott PJ, Wynne PM, Vine JH. Application of comprehensive two-dimensional gas chromatography to drugs analysis in doping control. J. Chromatogr. A. 2003;1000:109-24.

12. Poole CF. Solid-Phase Extraction. Elsevier, Amsterdam, the Netherlands 2020.

13. Seeley JV, Kramp FJ, Sharpe KS, Seeley SK. Characterization of gaseous mixtures of organic compounds with dual-secondary column comprehensive two-dimensional gas chromatography (GC $\times 2$ GC). J. Sep. Sci. 2002;25:53-9.

14. Libardoni M, Stevens PT, Waite JH, Sacks R. Analysis of human breath samples with a multi-bed sorption trap and comprehensive two-dimensional gas chromatography $(\mathrm{GC} \times \mathrm{GC})$. J. Chromatogr. B Anal. Technol. Biomed. Life Sci. 2006;842:13-21.

15. Mleth M, Schubert JK, Gröger T, Sabei B, Kischkel S, Fuchs P, Hein D, Zimmermann R, Miekisch W. Automated needle trap heart-cut GC/MS and needle trap comprehensive twodimensional GC/TOF-MS for breath gas analysis in the clinical environment. Anal. Chem. 2010;82:2541-51.

16. Risticevic S, DeEll JR, Pawliszyn J. Solid phase microextraction coupled with comprehensive two-dimensional gas chromatography-time-of-flight mass spectrometry for highresolution metabolite profiling in apples: Implementation of structured separations for optimization of sample preparation. J. Chromatogr. A. 2012;1251:208-18.

17. Cordero C, Cagliero C, Liberto E, Nicolotti L, Rubiolo P, Sgorbini B, Bicchi C. High concentration capacity sample preparation techniques to improve the informative potential of two-dimensional comprehensive gas chromatography-mass 
spectrometry: Application to sensomics. J. Chromatogr. A. 2013;1318:1-11.

18. Mondello L, Casilli A, Tranchida PQ, Dugo P, Dugo G. Detailed analysis and group-type separation of natural fats and oils using comprehensive two-dimensional gas chromatography. J. Chromatogr. A. 2003;1019:187-96.

19. Hyötyläinen T, Kallio M, Lehtonen M, Lintonen S, Peräjoki P, Jussila M, Riekkola ML. Comprehensive two-dimensional gas chromatography in the analysis of dietary fatty acids. J. Sep. Sci. 2004;27:459-67.

20. Welthagen W, Shellie RA, Spranger J, Ristow M, Zimmermann R, Fiehn O. Comprehensive two-dimensional gas chromatography-time-of-flight mass spectrometry $(\mathrm{GC} \times \mathrm{GC}$ TOF) for high resolution metabolomics: Biomarker discovery on spleen tissue extracts of obese NZO compared to lean C57BL/6 mice. Metabolomics. 2005;1:65-73.

21. Poole C, Mester Z, Miró M, Pedersen-Bjergaard S, Pawliszyn J. Glossary of terms used in extraction (IUPAC Recommendations 2016). Pure Appl. Chem. 2016;88:517-58.

22. Poole CF, Liquid-Phase Extraction. 2019.

23. Baltussen HA. New Concepts in Sorption Based Sample Preparation for Chromatography, Technische Universiteit Eindhoven, Eindhoven, The Netherlands, 2000.

24. Pawliszyn J. Comprehensive Sampling and Sample Preparation. Elsevier 2012.

25. Weggler BA, Gruber B, Teehan P, Jaramillo R, Dorman FL, in: Snow NHBT-SS, and T. (Ed.), Separation Science and Technology. Academic Press, New York, NY 2020, pp. 141-203.

26. Woolfenden E. Sorbent-based sampling methods for volatile and semi-volatile organic compounds in air. Part 2. Sorbent selection and other aspects of optimizing air monitoring methods. J. Chromatogr. A. 2010;1217:2685-94.

27. Prodhan MAI, Shi B, Song M, He L, Yuan F, Yin $X$, Bohman P, McClain CJ, Zhang X. Integrating comprehensive two-dimensional gas chromatography mass spectrometry and parallel two-dimensional liquid chromatography mass spectrometry for untargeted metabolomics. Analyst. 2019;144: 4331-41.

28. Fiehn O, Kopka J, Trethewey RN, Willmitzer L. Identification of uncommon plant metabolites based on calculation of elemental compositions using gas chromatography and quadrupole mass spectrometry. Anal. Chem. 2000;72:3573-80.

29. Amaral MSS, Nolvachai Y, Marriott PJ. Comprehensive TwoDimensional Gas Chromatography Advances in Technology and Applications: Biennial Update. Anal. Chem. 2020;92:85104.

30. Higgins Keppler EA, Jenkins CL, Davis TJ, Bean HD. Advances in the application of comprehensive two-dimensional gas chromatography in metabolomics. TrAC - Trends Anal. Chem. 2018;109:275-86.

31. Franchina FA, Mellors TR, Aliyeva M, Wagner J, Daphtary N, Lundblad LKA, Fortune SM, Rubin EJ, Hill JE. Towards the use of breath for detecting mycobacterial infection: A case study in a murine model. J. Breath Res. 2018;12:026008.

32. Mellors TR, Nasir M, Franchina FA, Smolinska A, Blanchet L, Flynn JL, Tomko J, O’Malley M, Scanga CA, Lin PL, Wagner J, Hill JE. Identification of Mycobacterium tuberculosis using volatile biomarkers in culture and exhaled breath. J. Breath Res. 2019;13:016004.
33. Schleich FN, Zanella D, Stefanuto PH, Bessonov K, Smolinska A, Dallinga JW, Henket M, Paulus V, Guissard F, Graff S, Moermans C, Wouters EFM, Van Steen K, Van Schooten FJ, Focant JF, Louis R. Exhaled volatile organic compounds are able to discriminate between neutrophilic and eosinophilic asthma. Am. J. Respir. Crit. Care Med. 2019;200:444-53.

34. Beccaria M, Bobak C, Maitshotlo B, Mellors TR, Purcaro G, Franchina FA, Rees CA, Nasir M, Black A, Hill JE. Exhaled human breath analysis in active pulmonary tuberculosis diagnostics by comprehensive gas chromatography-mass spectrometry and chemometric techniques. J. Breath Res. 2019;13: 016005.

35. Wilde MJ, Cordell RL, Salman D, Zhao B, Ibrahim W, Bryant L, Ruszkiewicz D, Singapuri A, Free RC, Gaillard EA, Beardsmore C, Thomas CLP, Brightling CE, Siddiqui S, Monks PS. Breath analysis by two-dimensional gas chromatography with dual flame ionisation and mass spectrometric detection - Method optimisation and integration within a large-scale clinical study. J. Chromatogr. A. 2019;1594:160-72.

36. Purcaro G, Nasir M, Franchina FA, Rees CA, Aliyeva M, Daphtary N, Wargo MJ, Lundblad LKA, Hill JE. Breath metabolome of mice infected with Pseudomonas aeruginosa. Metabolomics. 2019;15:10.

37. Gruber B, Keller S, Groeger T, Matuschek G, Szymczak W, Zimmermann R. Breath gas monitoring during a glucose challenge by a combined PTR-QMS/GC $\times$ GC-TOFMS approach for the verification of potential volatile biomarkers. J. Breath Res. 2016;10:036003.

38. Alonso M, Sanchez JM. Analytical challenges in breath analysis and its application to exposure monitoring. TrAC - Trends Anal. Chem. 2013;44:78-89.

39. Lawal O, Ahmed WM, Nijsen TME, Goodacre R, Fowler SJ. Exhaled breath analysis: a review of 'breath-taking' methods for off-line analysis. Metabolomics. 2017;13:1-16.

40. Zanella D, Focant JF, Hill JE, Stefanuto P, in: Beauchamp, D, Pleil JD. (Eds.), Breathborne Biomarkers and the Human Volatilome. Elsevierÿ Amsterdam, the Netherlands 2020, pp. 239-51.

41. Franchina FA, Zanella D, Dejong T, Focant J. Impact of the adsorbent material on volatile metabolites during in vitro and in vivo bio-sampling. Talanta. 2021;222:121569.

42. Franchina FA, Purcaro G, Burklund A, Beccaria M, Hill JE. Evaluation of different adsorbent materials for the untargeted and targeted bacterial VOC analysis using GC $\times$ GC-MS. Anal. Chim. Acta. 2019;1066:146-53.

43. Mack CI, Egert B, Liberto E, Weinert CH, Bub A, Hoffmann I, Bicchi C, Kulling SE, Cordero C. Robust Markers of Coffee Consumption Identified Among the Volatile Organic Compounds in Human Urine. Mol. Nutr. Food Res. 2019;63:1-12.

44. Loureiro CC, Oliveira AS, Santos M, Rudnitskaya A, Todo-Bom A, Bousquet J, Rocha SM. Urinary metabolomic profiling of asthmatics can be related to clinical characteristics. Allergy Eur. J. Allergy Clin. Immunol. 2016;71:1362-5.

45. Kim S, Yin X, Prodhan MAI, Zhang X, Zhong Z, Kato I. Global plasma profiling for colorectal cancer-associated volatile organic compounds: A proof-of-principle study. J. Chromatogr. Sci. 2019;57:385-96.

46. Eshima J, Ong S, Davis TJ, Miranda C, Krishnamurthy D, Nachtsheim A, Stufken J, Plaisier C, Fricks J, Bean HD, Smith 
BS. Monitoring changes in the healthy female metabolome across the menstrual cycle using GC $\times$ GC-TOFMS. J. Chromatogr. B Anal. Technol. Biomed. Life Sci. 2019;1121:48-57.

47. Bileck A, Verouti SN, Escher G, Vogt B, Groessl M. A comprehensive urinary steroid analysis strategy using two-dimensional gas chromatography-time of flight mass spectrometry. Analyst. 2018;143:4484-94.

48. Luier L, Loots DT. Tuberculosis metabolomics reveals adaptations of man and microbe in order to outcompete and survive. Metabolomics. 2016;12:1-9.

49. Luies L, Mienie J, Motshwane C, Ronacher K, Walzl G, Loots DT. Urinary metabolite markers characterizing tuberculosis treatment failure. Metabolomics. 2017;13:0.

50. Yu Z, Huang H, Reim A, Charles PD, Northage A, Jackson D, Parry I, Kessler BM. Optimizing 2D gas chromatography mass spectrometry for robust tissue, serum and urine metabolite profiling. Talanta. 2017;165:685-91.

51. Arslan FN, Kolk A, Janssen HG. Methods for one- and two-dimensional gas chromatography with flame ionization detection for identification of Mycobacterium tuberculosis in sputum. J. Chromatogr. B Anal. Technol. Biomed. Life Sci. 2019;124:204-17.

52. Di Giovanni N, Meuwis MA, Louis E, Focant JF. Untargeted Serum Metabolic Profiling by Comprehensive TwoDimensional Gas Chromatography-High-Resolution Time-ofFlight Mass Spectrometry. J. Proteome Res. 2020;19:1013-28.

53. Bressanello D, Liberto E, Collino M, Chiazza F, Mastrocola R, Reichenbach SE, Bicchi C, Cordero C. Combined untargeted and targeted fingerprinting by comprehensive twodimensional gas chromatography: revealing fructose-induced changes in mice urinary metabolic signatures. Anal. Bioanal. Chem. 2018;410:2723-37.

54. Misra BB, Bassey E, Bishop AC, Kusel DT, Cox LA, Olivier M. High-resolution gas chromatography/mass spectrometry metabolomics of non-human primate serum. Rapid Commun. Mass Spectrom. 2018;32:1497-506.

55. Watson NE, Parsons BA, Synovec RE. Performance evaluation of tile-based Fisher Ratio analysis using a benchmark yeast metabolome dataset. J. Chromatogr. A. 2016;1459:101-11.

56. Joseph JS, Ayeleso AO, Mukwevho E. Role of exercise-induced calmodulin protein kinase (CaMK)II activation in the regulation of omega- 6 fatty acids and lipid metabolism genes in rat skeletal muscle. Physiol. Res. 2017;66:969-77.

57. Roodt AP, Naudé Y, Stoltz A, Rohwer E. Human skin volatiles: Passive sampling and GC $\times$ GC-ToFMS analysis as a tool to investigate the skin microbiome and interactions with anthropophilic mosquito disease vectors. J. Chromatogr. B Anal. Technol. Biomed. Life Sci. 2018;1097-1098:83-93.

58. Doležal P, Kyjaková P, Valterová I, Urban Š. Qualitative analyses of less-volatile organic molecules from female skin scents by comprehensive two dimensional gas chromatography-time of flight mass spectrometry. J. Chromatogr. A. 2017;1505:77-86.

59. Rees CA, Nordick KV, Franchina FA, Lewis AE, Hirsch EB, Hill JE. Volatile metabolic diversity of Klebsiella pneumoniae in nutrient-replete conditions. Metabolomics. 2017; 13:18.

60. Purcaro G, Stefanuto PH, Franchina FA, Beccaria M, WielandAlter WF, Wright PF, Hill JE. SPME-GC $\times$ GC-TOF MS fingerprint of virally-infected cell culture: Sample preparation opti- mization and data processing evaluation. Anal. Chim. Acta. 2018;1027:158-67.

61. Mellors TR, Rees CA, Franchina FA, Burklund A, Patel C, Hathaway LJ, Hill JE. The volatile molecular profiles of seven Streptococcus pneumoniae serotypes. J. Chromatogr. B Anal. Technol. Biomed. Life Sci. 2018;1096:208-13.

62. Rees CA, Franchina FA, Nordick KV, Kim PJ, Hill JE. Expanding the Klebsiella pneumoniae volatile metabolome using advanced analytical instrumentation for the detection of novel metabolites. J. Appl. Microbiol. 2017;122:785-95.

63. Bean HD, Rees CA, Hill JE. Comparative analysis of the volatile metabolomes of Pseudomonas aeruginosa clinical isolates. J. Breath Res. 2016;10:047102.

64. Nasir M, Bean HD, Smolinska A, Rees CA, Zemanick ET, Hill JE. Volatile molecules from bronchoalveolar lavage fluid can "rule-in" Pseudomonas aeruginosa and "rule-out" Staphylococcus aureus infections in cystic fibrosis patients. Sci. Rep. 2018;8:826.

65. Zanella D, Henket M, Schleich F, Dejong T, Louis R, Focant JF, Stefanuto P-H. Comparison of the effect of chemically and biologically induced inflammation on the volatile metabolite production of lung epithelial cells by GC $\times$ GC-TOFMS. Analyst. 2020;145:5148-57.

66. Martins C, Brandão T, Almeida A, Rocha SM. Metabolomics strategy for the mapping of volatile exometabolome from Saccharomyces spp. widely used in the food industry based on comprehensive two-dimensional gas chromatography. J. Sep. Sci. 2017;40:2228-37.

67. Cardoso P, Santos M, Freitas R, Rocha SM, Figueira E. Response of Rhizobium to Cd exposure: A volatile perspective. Environ. Pollut. 2017;231:802-11.

68. Mousavi F, Gionfriddo E, Carasek E, Souza-Silva EA, Pawliszyn J. Coupling solid phase microextraction to complementary separation platforms for metabotyping of E. coli metabolome in response to natural antibacterial agents. Metabolomics. 2016;12:169.

69. Ebadzadsahrai G, Higgins Keppler EA, Soby SD, Bean HD. Inhibition of Fungal Growth and Induction of a Novel Volatilome in Response to Chromobacterium vaccinii Volatile Organic Compounds. Front. Microbiol. 2020;11:1035.

70. Miguez AM, McNerney MP, Styczynski MP. Metabolomics analysis of the toxic effects of the production of lycopene and its precursors. Front. Microbiol. 2018;9:1-15.

71. Fiehn O. Metabolomics - The link between genotypes and phenotypes. Plant Mol. Biol. 2002;48:155-71.

72. Liberto E, Bicchi C, Cagliero C, Cordero C, Rubiolo P, Sgorbini B. Food Chemistry, Function and Analysis. The Royal Society of Chemistry, London, UK 2020, pp. 3-37.

73. Paiva AC, Oliveira DS, Hantao LW. A bottom-up approach for data mining in bioaromatization of beers using flowmodulated comprehensive two-dimensional gas chromatography/mass spectrometry. Separations. 2019;6:46.

74. Stefanuto PH, Perrault KA, Dubois LM, L'Homme B, Allen C, Loughnane C, Ochiai N, Focant JF. Advanced method optimization for volatile aroma profiling of beer using twodimensional gas chromatography time-of-flight mass spectrometry. J. Chromatogr. A. 2017;1507:45-52.

75. Franchina FA, Zanella D, Lazzari E, Stefanuto PH, Focant JF. Investigating aroma diversity combining purge-and-trap, 
comprehensive two-dimensional gas chromatography, and mass spectrometry. J. Sep. Sci. 2020;43:1790-9.

76. Trujillo-Rodríguez MJ, Anderson JL. Silver-based polymeric ionic liquid sorbent coatings for solid-phase microextraction: Materials for the selective extraction of unsaturated compounds. Anal. Chim. Acta. 2019;1047:52-61.

77. Clark KD, Emaus MN, Varona M, Bowers AN, Anderson JL. Ionic liquids: solvents and sorbents in sample preparation. J. Sep. Sci. 2018;41:209-35.

78. Souza ID, Nan H, Queiroz MEC, Anderson JL. Tunable SilverContaining Stationary Phases for Multidimensional Gas Chromatography. Anal. Chem. 2019;91:4969-74.

79. Trujillo-Rodríguez MJ, Nan H, Varona M, Emaus MN, Souza ID, Anderson JL. Advances of Ionic Liquids in Analytical Chemistry. Anal. Chem. 2019;91:505-31.

80. Crucello J, Miron LFO, Ferreira VHC, Nan H, Marques MOM, Ritschel PS, Zanus MC, Anderson JL, Poppi RJ, Hantao LW. Characterization of the aroma profile of novel Brazilian wines by solid-phase microextraction using polymeric ionic liquid sorbent coatings. Anal. Bioanal. Chem. 2018;410:4749-62.

81. Gionfriddo E, Souza-Silva ÉA, Ho TD, Anderson JL, Pawliszyn J. Exploiting the tunable selectivity features of polymeric ionic liquid-based SPME sorbents in food analysis. Talanta. 2018;188:522-30.

82. Magagna F, Cordero C, Cagliero C, Liberto E, Rubiolo P, Sgorbini B, Bicchi C. Black tea volatiles fingerprinting by comprehensive two-dimensional gas chromatography - Mass spectrometry combined with high concentration capacity sample preparation techniques: Toward a fully automated sensomic assessment. Food Chem. 2017;225:276-87.

83. Chen Q, Zhu Y, Dai W, Lv H, Mu B, Li P, Tan J, Ni D, Lin Z. Aroma formation and dynamic changes during white tea processing. Food Chem. 2019;274:915-24.

84. Kang S, Yan H, Zhu Y, Liu X, Lv HP, Zhang Y, Dai WD, Guo L, Tan JF, Peng QH, Lin Z. Identification and quantification of key odorants in the world's four most famous black teas. Food Res. Int. 2019;121:73-83.

85. Shi J, Zhu Y, Zhang Y, Lin Z, Lv HP. Volatile composition of Fu-brick tea and Pu-erh tea analyzed by comprehensive two-dimensional gas chromatography-time-of-flight mass spectrometry. Lwt. 2019;103:27-33.

86. Stilo F, Tredici G, Bicchi C, Robbat A, Morimoto J, Cordero C. Climate and Processing Effects on Tea (Camellia sinensis L. Kuntze) Metabolome: Accurate Profiling and Fingerprinting by Comprehensive Two-Dimensional Gas Chromatography/Time-of-Flight Mass Spectrometry. Molecules. 2020;25:1-19.

87. Zhu Y, Lv HP, Shao CY, Kang S, Zhang Y, Guo L, Dai WD, Tan JF, Peng QH, Lin Z. Identification of key odorants responsible for chestnut-like aroma quality of green teas. Food Res. Int. 2018;108:74-82.

88. Novaes FJM, Junior S, da AI, Kulsing C, Nolvachai Y, Bizzo HR, Neto A, de FR, Rezende CM, Marriott PJ. New approaches to monitor semi-volatile organic compounds released during coffee roasting using flow-through/active sampling and comprehensive two-dimensional gas chromatography. Food Res. Int. 2019;119:349-58.

89. Novaes FJM, Kulsing C, Bizzo HR, de Aquino Neto FR, Rezende CM, Marriott PJ. Analysis of underivatised low volatil- ity compounds by comprehensive two-dimensional gas chromatography with a short primary column. J. Chromatogr. A. 2018;1536:75-81.

90. Cordero C, Guglielmetti A, Bicchi C, Liberto E, Baroux L, Merle $\mathrm{P}$, Tao Q, Reichenbach SE. Comprehensive two-dimensional gas chromatography coupled with time of flight mass spectrometry featuring tandem ionization: Challenges and opportunities for accurate fingerprinting studies. J. Chromatogr. A. 2019;1597:132-41.

91. Cialiè Rosso M, Liberto E, Spigolon N, Fontana M, Somenzi $\mathrm{M}$, Bicchi C, Cordero C. Evolution of potent odorants within the volatile metabolome of high-quality hazelnuts (Corylus avellana L.): evaluation by comprehensive two-dimensional gas chromatography coupled with mass spectrometry. Anal. Bioanal. Chem. 2018;410:3491-506.

92. Oliveira LF, Braga SCGN, Augusto F, Hashimoto JC, Efraim P, Poppi RJ. Differentiation of cocoa nibs from distinct origins using comprehensive two-dimensional gas chromatography and multivariate analysis. Food Res. Int. 2016;90:133-8.

93. Braga SCGN, Oliveira LF, Hashimoto JC, Gama MR, Efraim P, Poppi RJ, Augusto F. Study of volatile profile in cocoa nibs, cocoa liquor and chocolate on production process using GC $\times$ GC-QMS. Microchem. J. 2018;141:353-61.

94. Magagna F, Liberto E, Reichenbach SE, Tao Q, Carretta A, Cobelli L, Giardina M, Bicchi C, Cordero C. Advanced fingerprinting of high-quality cocoa: Challenges in transferring methods from thermal to differential-flow modulated comprehensive two dimensional gas chromatography. J. Chromatogr. A. 2018;1536:122-36.

95. Lukić I, Carlin S, Horvat I, Vrhovsek U. Combined targeted and untargeted profiling of volatile aroma compounds with comprehensive two-dimensional gas chromatography for differentiation of virgin olive oils according to variety and geographical origin. Food Chem. 2019;270:403-14.

96. Stilo F, Cordero C, Sgorbini B, Bicchi C, Liberto E. Highly informative fingerprinting of extra-virgin olive oil volatiles: The role of high concentration-capacity sampling in combination with comprehensive two-dimensional gas chromatography. Separations. 2019;6:34.

97. Aloisi I, Zoccali M, Dugo P, Tranchida PQ, Mondello L. Fingerprinting of the unsaponifiable fraction of vegetable oils by using cryogenically-modulated comprehensive two-dimensional gas chromatography-high resolution time-of-flight mass spectrometry. Food Anal. Methods. 2020;13:1523-9.

98. Barp L, Franchina FA, Purcaro G, Tranchida PQ, Mondello L. In-pipette solid-phase extraction prior to flow-modulation comprehensive two-dimensional gas chromatography with dual detection for the determination of minor components in vegetable oils. Talanta. 2017;165:598-603.

99. Risticevic S, Souza-Silva EA, DeEll JR, Cochran J, Pawliszyn J. Capturing Plant Metabolome with Direct-Immersion in Vivo Solid Phase Microextraction of Plant Tissues. Anal. Chem. 2016;88:1266-74.

100. Risticevic S, Souza-Silva EA, Gionfriddo E, DeEll JR, Cochran J, Hopkins WS, Pawliszyn J. Application of in vivo solid phase microextraction (SPME) in capturing metabolome of apple (Malus ×domestica Borkh.) fruit. Sci. Rep. 2020;10:1-11.

101. Zhu G, Liu F, Li P, He S, Zhu S, Gao Q, Feng Y. Profiling free fatty acids in edible oils via magnetic dispersive extrac- 
tion and comprehensive two-dimensional gas chromatographymass spectrometry. Food Chem. 2019;297:124998.

102. Qiu X, Cao L, Han R. Analysis of volatile components in different ophiocordyceps sinensis and insect host products. Molecules. 2020;25:1603.

103. Vaníčková L, Pompeiano A, Maděra P, Massad TJ, Vahalík P. Terpenoid profiles of resin in the genus Dracaena are species specific. Phytochemistry. 2020;170:112197.

104. Jiang M, Kulsing C, Marriott PJ. Comprehensive 2D gas chromatography-time-of-flight mass spectrometry with $2 \mathrm{D}$ retention indices for analysis of volatile compounds in frankincense (Boswellia papyrifera). Anal. Bioanal. Chem. 2018;410:3185-96.

105. Graves BM, Johnson TJ, Nishida RT, Dias RP, Savareear B, Harynuk JJ, Kazemimanesh M, Olfert JS, Boies AM. Comprehensive characterization of mainstream marijuana and tobacco smoke. Sci. Rep. 2020;10:7160.

106. Marchini M, Charvoz C, Dujourdy L, Baldovini N, Filippi JJ. Multidimensional analysis of cannabis volatile constituents: Identification of 5,5-dimethyl-1-vinylbicyclo[2.1.1] hexane as a volatile marker of hashish, the resin of Cannabis sativa L. J. Chromatogr. A. 2014;1370:200-15.

107. Franchina F, Dubois L, Focant J-F. In-depth cannabis multiclass metabolite profiling using sorptive extraction and multidimensional gas chromatography with low- and high-resolution mass spectrometry. Anal. Chem. 2020;92:10512-20.

108. Carrero-Carralero C, Rodríguez-Sánchez S, Calvillo I, Martínez-Castro I, Soria AC, Ramos L, Sanz ML. Gas chromatographic-based techniques for the characterization of low molecular weight carbohydrates and phenylalkanoid glycosides of Sedum roseum root supplements. J. Chromatogr. A. 2018;1570:116-25.

109. Yan DD, Wong YF, Tedone L, Shellie RA, Marriott PJ, Whittock SP, Koutoulis A. Chemotyping of new hop (Humulus lupulus L.) genotypes using comprehensive two-dimensional gas chromatography with quadrupole accurate mass time-of-flight mass spectrometry. J. Chromatogr. A. 2018;1536:110-21.

110. Bendif H, Adouni K, Miara MD, Baranauskienè R, Kraujalis P, Venskutonis PR, Nabavi SM, Maggi F. Essential oils (EOs), pressurized liquid extracts (PLE) and carbon dioxide supercritical fluid extracts (SFE-CO2) from Algerian Thymus munbyanus as valuable sources of antioxidants to be used on an industrial level. Food Chem. 2018;260:289-98.

111. Tissandié L, Brevard H, Belhassen E, Alberola M, Meierhenrich U, Filippi JJ. Integrated comprehensive two-dimensional gas-chromatographic and spectroscopic characterization of vetiveryl acetates: Molecular identifications, quantification of constituents, regulatory and olfactory considerations. J. Chromatogr. A. 2018;1573:125-50.

112. Zoccali M, Tranchida PQ, Bonaccorsi IL, Dugo P, Mondello L, Dugo G. Detailed profiling of the volatile oxygenated fraction of mandarin essential oils by using the off-line combination of high-performance liquid chromatography and comprehensive two-dimensional gas chromatography-mass spectrometry. Food Anal. Methods. 2017;10:1106-16.

113. Zoccali M, Giocastro B, Bonaccorsi IL, Trozzi A, Tranchida $\mathrm{PQ}$, Mondello L. In-depth qualitative analysis of lime essential oils using the off-line combination of normal phase high performance liquid chromatography and comprehensive twodimensional gas chromatography-quadrupole mass spectrometry. Foods. 2019;8:580.

114. Yan DD, Wong YF, Whittock SP, Koutoulis A, Shellie RA, Marriott PJ. Sequential Hybrid Three-Dimensional Gas Chromatography with Accurate Mass Spectrometry: A Novel Tool for High-Resolution Characterization of Multicomponent Samples. Anal. Chem. 2018;90:5264-71.

115. De Grazia S, Gionfriddo E, Pawliszyn J. A new and efficient Solid Phase Microextraction approach for analysis of high fat content food samples using a matrix-compatible coating. Talanta. 2017;167:754-60.

116. Psillakis E. The effect of vacuum: an emerging experimental parameter to consider during headspace microextraction sampling. Anal. Bioanal. Chem. 2020;412:5989-97.

117. Emmons RV, Tajali R, Gionfriddo E. Development, optimization and applications of thin film solid phase microextraction (TF-SPME) devices for thermal desorption: A comprehensive review. Separations. 2019;6:39.

118. Díaz-Liñán MC, García-Valverde MT, Lucena R, Cárdenas S, López-Lorente AI. Paper-based sorptive phases for microextraction and sensing. Anal. Methods. 2020;12:3074-91.

119. Ochiai N, Sasamoto K, David F, Sandra P. Recent Developments of Stir Bar Sorptive Extraction for Food Applications: Extension to Polar Solutes. J. Agric. Food Chem. 2018;66:7249-55.

\section{SUPPORTING INFORMATION}

Additional supporting information may be found online in the Supporting Information section at the end of the article.

How to cite this article: Franchina FA, Zanella D, Dubois LM, Focant J. The role of sample preparation in multidimensional gas chromatographic separations for non-targeted analysis with the focus on recent biomedical, food, and plant applications. J Sep Sci. 2021;44:188-210. https://doi.org/10.1002/jssc.202000855 